\title{
Recommended Instruments for Analyzing Cyber Dating Violence: A Systematic Review
}

\author{
Ainize Martínez Soto and Izaskun Ibabe $\mathbb{D}$ \\ Universidad del País Vasco (Spain)
}

\begin{abstract}
Cyber dating violence is an emerging form of dating violence that may have serious health effects on adolescents and young people, and in recent years interest in its study has increased. In order to understand completely the nature and magnitude of the problem, a clear understanding of the concept, constructs and well-established measurement tools are needed. The goal of this study was to analyze the measurement instruments of cyber dating violence in adolescents and young adults, and to determine which are the best suitable to use. To accomplish these objectives a systematic review was carried out. After reviewing the literature, twenty-four measurement instruments were analyzed, with important differences found between them in terms, constructs, dimensions and measurement attributes, as well as differences in their assessed psychometric properties. Once the methodological quality evaluation of the instruments was carried out following COSMIN (COnsensus based Standards for the selection of health Measurement Instruments) guidelines, three scales were found to be recommendable depending on the age and cultural context of participants: Cyber Dating Abuse Questionnaire (Borrajo, Gámez-Guadix, Pereda, et al., 2015), Technology-facilitated Abuse in Relationships Scale (Brown \& Hegarty, 2021), and Abuse in Teen Relationships (CARPA; Calvete et al., 2021).
\end{abstract}

Received 5 May 2021; Revised 16 December 2021; Accepted 20 December 2021

Keywords: cyber dating abuse, cyber dating violence, instruments, technology, youth

Young people's use of Information and Communication Technologies (ICTs) to communicate and interact with other people has increased in recent decades (Duerksen \& Woodin, 2019). Technologies have changed the ways in which young people relate to each other, including dating relationship dynamics. Burke et al. (2011) found that young people use a large number of media and digital tools to develop and maintain dating relationships (e.g., text messages, emails, mobile phones, social networks, or webcams). It is undeniable that the use of ICTs can have important benefits on personal and social levels, as well as positive effects on adolescents' socialization (Caridade et al., 2019), allowing them to explore and define their identity and personality, express themselves and build new relationships (Hinduja \& Patchin, 2020). However, these tools may also

Correspondence concerning this article should be addressed to Izaskun Ibabe Erostarbe. Universidad del País Vasco. Departamento de Psicología Clínica y de la Salud y Metodología de Investigación. Campus Gipuzkoa. 20018 Donostia (Spain).

E-mail: izaskun.ibabe@ehu.eus

Funding Statement: This research received no specific grant from any funding agency, commercial or not-for-profit sectors.

Conflicts of Interest: None. provide new opportunities for some individuals to exert control over others, given that it is now easier than ever before to stalk someone, collect information on them as well as harass them in multiple contexts (Fernet et al., 2019). Technology has modified the ways in which violence can be perpetrated and suffered, making it immediate, beyond any physical limit, through a wide number of media (e.g., email, instant messaging services and social networks) and with minimal effort, thereby causing a greater impact on the victim, more rapidly and in different areas of his/her life. In this way, along with these changes in relationships, a new form of intimate partner violence has also emerged, called cyber or digital dating violence or abuse (Zweig et al., 2014).

The range of terminology used in the scientific community is currently very wide and varied, probably since it is a relatively recent phenomenon. In the absence of a definitive term, this review proposes that the term to

How to cite this article:

Martínez Soto, A., \& Ibabe, I. (2022). Recommended instruments for analyzing cyber dating violence: A systematic review. The Spanish Journal of Psychology, 25. e4. Doi:10.1017/SJP.2021.50 
use in future studies should be cyber dating violence (CDV) because, on the one hand, the term cyber denotes a relationship with information technology (encompassing all kinds of technologies relates to the virtual world) and, on the other hand, the term dating violence refers to violence in relationships between adolescents or young people, where the members of the couple do not live together, are not financially independent from their parents, and do not have well-established relationships (Ibabe et al., 2020). Cyber dating violence has been defined as a form of control and harassment by the dating partner or ex-partner using new technologies and the media (Zweig et al., 2014). It has also been observed that the definitions of CDV tend to include a list of possible behaviors and different technological media, which is not wholly adequate for clarifying the construct. Rodríguez-Domínguez et al. (2020) pointed out methodological deficiencies in the construction of knowledge in the field of CDV, indicating for example, a lack of attention to manifestations of sexual aggression. Although it is not easy to establish a definitive classification of the different manifestations of CDV due to emerging new behaviors related to technology use, we propose five dimensions. Thus, cyber dating violence is understood in this paper as the perpetration of different types of aggression (psychological direct, control, public harassment, exclusion, and sexual) to the partner or ex-partner in the context of a dating or courtship relationship, via any digital media. Table 1 shows the definitions and examples of the five potential dimensions of the cyber dating violence construct.

To advance research on CDV, it is important not only to have an appropriate conceptualization of CDV but also adequate instruments for its measurement (Cava \& Buelga, 2018). Consequently, the objective of this research was to describe the assessment instruments most used in CDV among young couples and to identify the best based on the quality of evidence in their psychometric properties. To achieve this goal, a systematic review was carried out, ensuring transparent and complete reporting of the systematic search. This systematic review can be helpful in understanding the reasons for the differences found in results regarding CDV prevalence rates in all previous reviews (Brown \& Hegarty, 2018; Caridade et al., 2019; Cavalcanti \& Coutinho, 2019; Rodríguez-Domínguez et al., 2020) since it shows how all the instruments present different conditions in their methodological approaches, which can affect the interpretation of the evidence found in their research. The present review analyzed the quality of psychometric properties using the COSMIN checklist, which takes into account both the methodological quality and results of each psychometric property.

\section{Method}

\section{Systematic Review}

This systematic literature review was conducted according to the Preferred Reporting Items for Systematic Reviews and Meta-analyses (PRISMA) guidelines with a 27-item checklist in order to ensure transparent reporting (Page et al., 2021). The identification, screening, and eligibility of the studies included are outlined in the flow diagram (see Figure 1).

\section{Search Strategies}

The bibliographical searches were carried out in three electronic databases: Web of Science (title, abstract, author keyword and keyword plus), Scopus (title, abstract, author keyword), and PsycARTICLES (all text option). Journal articles published in English, Spanish or Portuguese up to July 2021 were selected. During the full-text assessment for eligibility, the reference lists of the selected articles were also reviewed to identify additional studies that were not identified during the bibliographical search. Retrieved articles from three databases were exported to RefWorks in order to make the removal of duplicates much easier. The selection process was carried out by reading abstracts, but sometimes articles were removed after reading the full text.

For the search, 27 terms of CDV were used ("electronic victimization" OR "cyber dating abuse" OR "cyber dating aggression" OR "cyber dating victimization" OR "cyber intimate partner violence" OR "ciberviolencia de pareja" OR "cyber dating violence" OR "ciberviolencia en el noviazgo" OR "digital dating abuse" OR "digital dating violence" OR "digital dating victimization" OR "digital dating aggression" OR "intimate partner violence through electronic mediums" OR "violencia de pareja a través de medios electrónicos" OR "computer mediated communication based teen dating violence" OR "cyber psychological abuse in romantic relationships" OR "intimate partner cyber aggression victimization" OR "virtual intimate partner violence" OR "violencia de pareja online en la adolescencia" OR "violencia de pareja virtual" OR "electronic aggression in emerging adult romantic relationships" OR "electronic intrusion cyber dating aggression" OR "ciberagresión en el noviazgo" OR "electronic dating aggression" OR "technology assisted adolescent violence" OR "technological intimate partner violence" OR "partner directed cyber aggression").

\section{Eligibility Criteria}

For this review, the following inclusion criteria were applied: (a) Publications that adopt constructs strictly related to the phenomenon of cyber dating violence 
Table 1. Terms, Definitions and Examples of Behaviors of Each Dimension of Cyber Dating Violence

\begin{tabular}{|c|c|c|}
\hline $\begin{array}{l}\text { Term of each } \\
\text { dimension }\end{array}$ & Definitions & Examples \\
\hline $\begin{array}{l}\text { A. Cyber psycho- } \\
\text { logical aggres- } \\
\text { sion }\end{array}$ & $\begin{array}{l}\text { Behaviors that are intended to cause harm to } \\
\text { the partner, directly insulting or saying unpleasant things } \\
\text { or threatening to hurt in a private sphere using technology of } \\
\text { information. }\end{array}$ & $\begin{array}{l}\text { - Sending insulting and/or demean- } \\
\text { ing messages. } \\
\text { - Using capital letters to shout. }\end{array}$ \\
\hline B. Cyber control & $\begin{array}{l}\text { Use of electronic means to control the partner/ex-partner, } \\
\text { including behaviors related to surveillance or the invasion of } \\
\text { privacy of the partner. }\end{array}$ & $\begin{array}{l}\text { - Checking where and with whom } \\
\text { the partner is. } \\
\text { - Reading messages without per- } \\
\text { mission. } \\
\text { - Excessive control behaviors } \\
\text { on social networks. }\end{array}$ \\
\hline $\begin{array}{l}\text { C. Public harass- } \\
\text { ment }\end{array}$ & $\begin{array}{l}\text { Publishing threatening, insulting, or harmful messages through } \\
\text { social networks, spreading rumors about the partner, showing } \\
\text { private or embarrassing photos/videos to humiliate or embarrass } \\
\text { victim. }\end{array}$ & $\begin{array}{l}\text { - Publishing inappropriate photos of } \\
\text { the partner without permission. } \\
\text { - Dissemination compromising } \\
\text { information. }\end{array}$ \\
\hline D. Exclusion & Removing, excluding, or blocking on social networks or friend lists. & $\begin{array}{l}\text { - Block on a Web site such as Face- } \\
\text { book. } \\
\text { - Remove from WhatsApp list. } \\
\text { - Exclude from top friend list. }\end{array}$ \\
\hline $\begin{array}{c}\text { E. Cyber sexual } \\
\text { aggression }\end{array}$ & $\begin{array}{l}\text { Pressuring and threatening partners to have sex with him/her or do } \\
\text { sexual things in person or virtual when he/she knew the partner } \\
\text { did not want to. }\end{array}$ & $\begin{array}{l}\text { - Threaten to distribute sexual } \\
\text { images to have sex. } \\
\text { - Unwanted sexual things online. }\end{array}$ \\
\hline
\end{tabular}

(e.g., dating partners); (b) studies on the adolescent and/or young population (with a maximum mean age of 28-30 years or slightly higher); (c) publications in English, Spanish and Portuguese. The first criterion is related to the fact that some studies analyze this phenomenon through constructs in nonintimate relationships such as cyberbullying (e.g., cyber aggression, cyber violence).

The exclusion criteria applied were: (a) Studies analyzing cyber abuse in nonintimate relationships (e.g., between peers); (b) theoretical articles or qualitative studies; (c) studies not including cyber dating violence measures; (d) studies on a mostly adult population; and (e) studies focused on single behavior of cyber dating violence (e.g., electronic intrusion, sexting coercion). This last criterion is related to the fact that some publications only analyze single behavioral dimensions. Since cyber dating violence is conceptualized as multidimensional, only studies that explored this phenomenon's multidimensionality were included. Figure 1 shows the literature search flowchart and the selection of articles from the analyzed sources, with a total of 78 scientific articles addressing CDV obtained. Table 2 lists all psychometric studies using the instruments. Moreover, the remaining studies (which used CDV instruments but were not psychometric studies) are presented in Appendix A. All studies have the corresponding reference list, but the psychometric studies analyzed in the paper have an asterisk in the References section.

\section{Analysis of the Psychometric Evidence of the Instruments}

Some of the standards developed in the COSMIN Guide (COnsensus based Standards for the selection of health Measurement INstruments) were used to evaluate the psychometric properties of the analyzed instruments (Mokkink et al., 2018) following three steps:

First step: Assessment of the methodological quality of the studies. The COSMIN Risk of Bias checklist is applied. The term "risk of bias" refers to whether the results, based on the methodological quality of each study, are trustworthy. This checklist contains different standards referring to design requirements of studies on measurement properties. For each measurement property a COSMIN box is developed, containing different items, named standards, which are needed to assess the quality of a study on that specific measurement property. For example, Standard 1 on Structural Validity indicates that to determine the structure of the instrument a Confirmatory Factor Analysis (Rating: Very good) is preferred over Explorative Factor Analysis (Rating: Adequate). COSMIN Risk of Bias checklist contains ten boxes with standards for: (a) Instrument development (35 items), (b) content validity (31 items), (c) structural validity (4 items), (d) internal consistency 


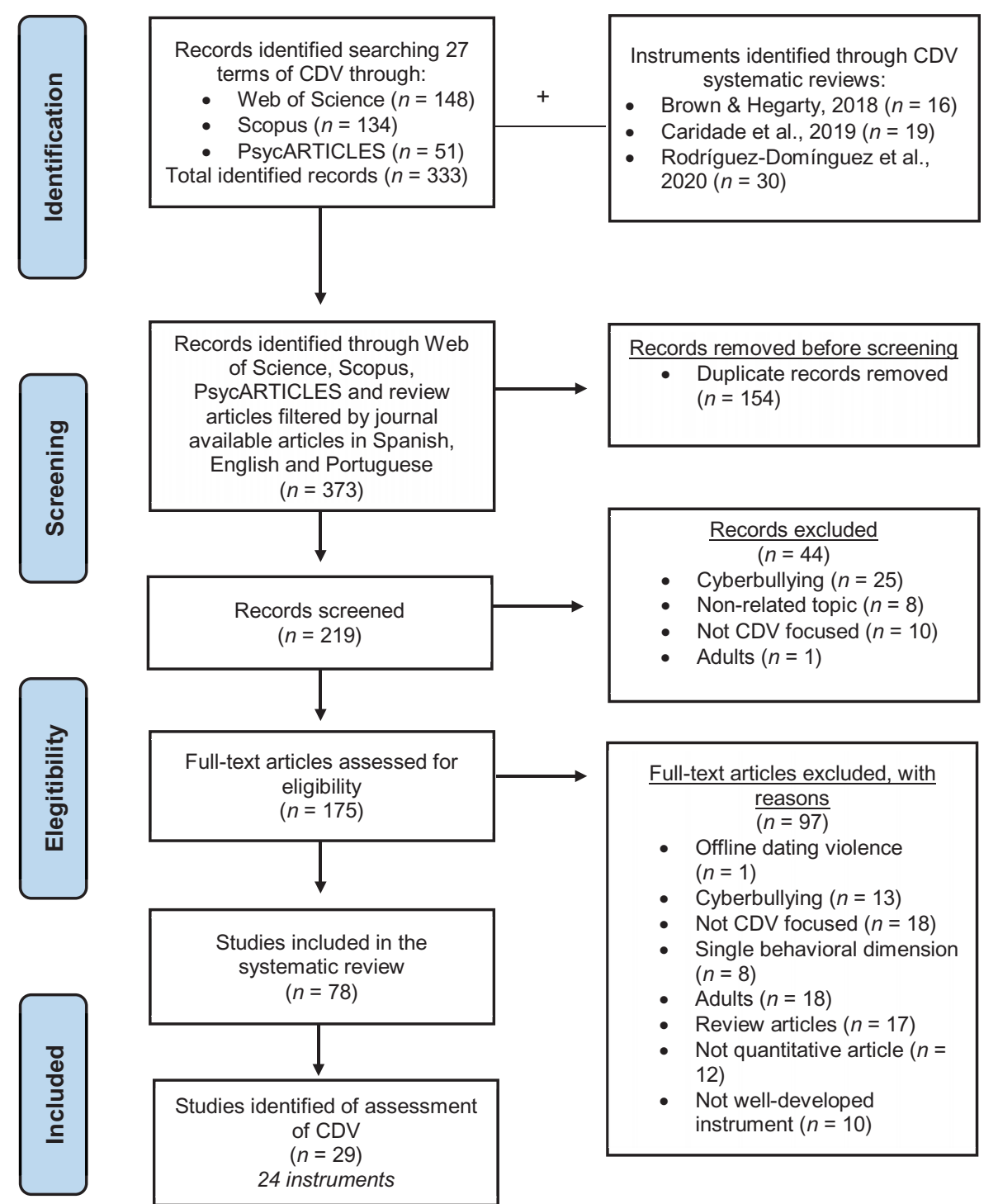

Figure 1. Flowchart of the Review Process according to Preferred Reporting Items for Systematic Reviews and Meta-Analyses

(5 items), (e) cross-cultural validity (4 items), (f) reliability (8 items), (g) measurement error (6 items), (h) criterion validity ( 3 items), (i) hypothesis testing for construct validity (7 items), and (j) responsiveness (13 items). The evaluation of these properties was achieved by rating 116 items on a four-point scale (very good, adequate, doubtful, inadequate quality, or not applicable). An overall rating for methodological quality was provided for each reported psychometric property by taking the lowest rating of any standard in the box (i.e., "the worst score counts" principle).

Second step: Assessment of the reported psychometric properties quality. The results of eight psychometric properties (content validity, structural validity, internal consistency, cross-cultural validity, reliability, criterion validity, hypothesis testing, and responsiveness) should be based on the results of all available studies per measurement property in order to determine whether each measurement property of the instrument is sufficient $(+)$ within the pre-established quality criteria, insufficient (-) outside the preestablished quality criteria, inconsistent $( \pm)$, or indeterminate (?) or less rigorous evidence or procedures that did not meet the pre-established quality criteria (Terwee et al., 2007). Additional details on these criteria can be found in the COSMIN manual for systematic reviews of instruments (Mokkink et al., 2018) (See Appendix B).

Third step: Best evidence synthesis: the GRADE approach. The quality of the overall evidence of each category was rated on a four-point scale (high, moderate, low, or very low), and instruments were categorized on the overall quality of evidence and results: Category A (instruments in this category will be recommended for 
use because results obtained with this instrument can be trusted), Category B (instruments may be used with caution, but require further research) and Category $\mathrm{C}$ (use of the measures in this category is not recommended). In cases classified as indeterminate or inconsistent it was not possible to assess quality with the GRADE approach. The recommended instruments which are useful and valid for measuring the same construct are those with higher COSMIN scores.

Table 2 shows the characteristics of the selected instruments and psychometric studies in this systematic review. The results of each study and instrument with respect to methodological quality and reported psychometric properties quality rating are shown in Table 3. A trained researcher analyzed the methodological quality and psychometric properties quality of 29 studies. However, the best evidence synthesis was done by consensus between two trained researchers, specifically, the overall assessment of each instrument (total sufficient rating, quality of evidence and category).

\section{Results}

The search strategy resulted in 333 articles retrieved from three different databases and other systematic reviews. From this, 154 duplicates were found, resulting in 219 articles as shown in Figure 1. After assessing articles for inclusion and exclusion criteria, a total of 24 instruments and 29 psychometric studies were included in the methodological evaluation using the COSMIN Risk of Bias checklist.

\section{General Characteristics of the Selected Instruments}

In Table, 2 characteristics of the psychometric studies corresponding to 24 instruments are shown. Most of the studies were conducted in the USA $(n=12)$, followed by Spain $(n=7)$, while the remaining studies were distributed in different countries (Mexico, England, Australia, Canada, Chile or Portugal). Important differences among the instruments in the evaluated aspects (types of digital media, perspective -perpetration vs. victimization, and time frame) were found. Moreover, the dimensions of CDV analyzed in these studies were very varied. In some studies, the CDV construct was underrepresented by focusing on measuring specific types of cyber dating violence (Rodríguez-Domínguez et al., 2020). Control behaviors, cyber psychological abuse and harassment were most commonly studied. It seems that there was no consensus regarding the construct of CDV, and the variations in dimensions across instruments hinder cross-study comparison. According to the categorization defended in the present study, the dimensions less taken into account in the analyzed studies were exclusion and sexual aggression.

\section{Synthesized Evidence}

The overall ratings of the evidence for each measurement property of each instrument and their risk of bias ratings are reported in Table 3. No studies assessed or showed evidence of cross-cultural validity, test-retest reliability, criterion validity or responsiveness, so these properties are not discussed nor included in the table.

\section{Content Validity}

Content validity is considered to be the most important measurement property of an instrument; this refers to the relevance, comprehensiveness, and comprehensibility of the instrument, that is, the degree to which the content is an adequate reflection of the construct to be measured (Terwee et al., 2018). Just one of the 29 studies performed a content validity study besides the development study and was thus the only one to receive a "high" rating on this property. Thirteen instruments did not even have development studies, having only the reviewer rating, thus receiving a "very low" rating. The remaining studies obtained a "moderate" rating, as they had performed a development study for the instrument. Knowing that content validity is the most important measurement property and one which can affect all others, the number of studies with very low ratings in this property is surprisingly high.

\section{Structural Validity}

Structural validity is a relevant measure when the instrument is based on a reflective model, in which all items are a manifestation of the same underlying construct (Mokkink et al., 2018). Seventeen of the twentynine studies examined the structural validity of the analyzed instruments. Thirteen studies conducted a confirmatory factor analysis and so received a "very good" rating for their methodological quality, while four performed just an exploratory factor analysis, thus being considered "adequate". The remaining studies did not provide any information regarding this property.

\section{Internal Consistency}

Internal consistency measures the degree of the interrelatedness among the items, that is, the extent to which the items in a subscale are measuring the same concept. This property is relevant for questionnaires measuring a single construct by using multiple items (Terwee et al., 2007). Twenty-eight studies assessed the internal consistency of the instruments, of which five were considered "inadequate". These ratings were due to the internal consistency statistics being calculated for the entire scale, not each subscale separately. One was "doubtful" due to lack of evidence that the scale was 
Table 2. Sample Characteristics, Dimensions, Digital Media, Perspective and Time Frame of the Selected Instruments

\begin{tabular}{|c|c|c|c|c|c|}
\hline Instrument/Source & $\begin{array}{l}\text { Num. } \\
\text { study }\end{array}$ & $\begin{array}{l}\text { Sample characteristics (of } \\
\text { the original validation study } \\
\text { and other studies) }\end{array}$ & $\begin{array}{l}\text { Dimensions (Items) } \\
\text { (Cat.) }\end{array}$ & Scope of digital media & $\begin{array}{l}\text { Perspective/Time } \\
\text { frame }\end{array}$ \\
\hline $\begin{array}{l}\text { 1. Electronic Victimization (EV) } \\
\text { Bennett et al. (2011) }\end{array}$ & 2 & $\begin{array}{l}n=403 ; 68 \% \text { female } \\
\text { Age }=18-22 \text { years old USA }\end{array}$ & $\begin{array}{l}\text { Hostility }(7)(A, C) \\
\text { Intrusion (7) (B) } \\
\text { Humiliation (5) (A,C) } \\
\text { Exclusion (3) (D) }\end{array}$ & $\begin{array}{l}\text { Text; E-mail; Social networking sites; Internet; } \\
\text { Chat room; Mobile phone; Instant messaging; } \\
\text { Photos }\end{array}$ & $\begin{array}{l}\text { Victimization in the last } \\
\text { year }\end{array}$ \\
\hline $\begin{array}{l}\text { 2. Cyber Dating Abuse Questionnaire } \\
\text { (CDAQ-A) } \\
\text { Borrajo, Gámez-Guadix, \& Calvete } \\
(2015 \mathrm{~b})\end{array}$ & 1 & $\begin{array}{c}n=433 ; 60 \% \text { female; Age }= \\
18-30 \text { years old; Spain }\end{array}$ & $\begin{array}{l}\text { Dimensions not } \\
\text { identified }(9)(A, B, C)\end{array}$ & $\begin{array}{l}\text { Social networking sites; Instant messaging; } \\
\text { E-mail }\end{array}$ & $\begin{array}{l}\text { Victimization not } \\
\text { indicated }\end{array}$ \\
\hline $\begin{array}{l}\text { 3. Cyber Dating Abuse Questionnaire } \\
\text { (CDAQ-B) } \\
\text { Study 1: Borrajo, Gámez-Guadix, } \\
\text { Pereda et al. (2015) } \\
\text { Study 2: Caridade \& Braga (2019) } \\
\text { Study 3: Hidalgo-Rasmussen et al. } \\
\quad \text { (2020) } \\
\text { Study 4: Lara (2020) }\end{array}$ & 23 & $\begin{array}{l}n=788 ; 77 \% \text { female } \\
\text { Age }=18-30 \text { years old; Spain } \\
n=272 ; 87.1 \% \text { female; Age }= \\
\quad 28.41 \text { years old; Portugal } \\
n=534 ; 51.6 \% \text { female } \\
\text { Age }=14.6 \text { year; Mexico } \\
n=1,538 ; 59.8 \% \text { female }\end{array}$ & $\begin{array}{l}\text { Direct aggression (11) } \\
(\mathrm{A}, \mathrm{C}) \\
\text { Control/monitoring } \\
\text { (9) (B) }\end{array}$ & $\begin{array}{l}\text { Social networking sites; New technologies; } \\
\text { E-mail; Mobile phone; Camera; Video }\end{array}$ & $\begin{array}{l}\text { Victimization } \\
\text { perpetration in the } \\
\text { last year }\end{array}$ \\
\hline $\begin{array}{l}\text { 4. Technology-facilitated Abuse in } \\
\text { Relationships Scale (TAR) } \\
\text { Brown \& Hegarty (2021) }\end{array}$ & 2 & $\begin{array}{l}\text { Age }=14-24 \text { years old; Chile } \\
n=555 ; 52.8 \% \text { female; Age }= \\
\quad 16-24 \\
\text { Australia }\end{array}$ & $\begin{array}{l}\text { Humiliation }(10)(\mathrm{A}, \mathrm{C}) \\
\text { Monitoring \& Control } \\
\quad(7)(\mathrm{B}) \\
\text { Sexual coercion }(8)(\mathrm{E}) \\
\text { Threats (5) (A) }\end{array}$ & $\begin{array}{l}\text { Web; Images, Digital device, Video; Online } \\
\text { profile; Digital conversation; Tracking } \\
\text { software; Phone; Live video; Message; Calls }\end{array}$ & $\begin{array}{l}\text { Victimization } \\
\text { perpetrationv in the } \\
\text { last } 12 \text { months }\end{array}$ \\
\hline $\begin{array}{l}\text { 5. Controlling Partner Inventory (CPI) } \\
\text { Burke et al. (2011) }\end{array}$ & 2 & $\begin{array}{c}n=804 ; 67 \% \text { female; Age }= \\
18-23 \text { years old; USA }\end{array}$ & $\begin{array}{l}\text { Photos/camera/GPS/ } \\
\text { Spyware (7) (B) } \\
\text { Excessive } \\
\text { communication } \\
\text { (4) (B) } \\
\text { Threatening behaviors } \\
\text { (3) (A) } \\
\text { Checking behaviors } \\
\text { (4) (B) }\end{array}$ & $\begin{array}{l}\text { E-mail; Mobile phone; Text; Social networking } \\
\text { sites; Photos; Global Positioning System; Web } \\
\text { cam }\end{array}$ & $\begin{array}{l}\text { Victimization } \\
\text { perpetration ever }\end{array}$ \\
\hline
\end{tabular}


Table 2. Continued.

\begin{tabular}{|c|c|c|c|c|c|}
\hline Instrument/Source & $\begin{array}{l}\text { Num. } \\
\text { study }\end{array}$ & $\begin{array}{l}\text { Sample characteristics (of } \\
\text { the original validation study } \\
\text { and other studies) }\end{array}$ & $\begin{array}{l}\text { Dimensions (Items) } \\
\text { (Cat.) }\end{array}$ & Scope of digital media & $\begin{array}{l}\text { Perspective/Time } \\
\text { frame }\end{array}$ \\
\hline $\begin{array}{l}\text { 6. Adolescent Relationship Abuse } \\
\text { Questionnaire (CARPA) } \\
\text { Calvete et al. (2021) }\end{array}$ & 3 & $\begin{array}{l}n=886 ; 51.7 \% \text { female } \\
\text { Age }=11-18 \text { years old } \\
\text { Spain }\end{array}$ & $\begin{array}{l}\text { Online direct } \\
\text { aggression (8) } \\
(\mathrm{A}, \mathrm{C}, \mathrm{E}) \\
\text { Online control (3) (B) }\end{array}$ & $\begin{array}{l}\text { Mobile; Internet; Social Networking Site; } \\
\text { Messages; Photos; Videos; Mail; Calls }\end{array}$ & $\begin{array}{l}\text { Victimization } \\
\text { perpetration in the } \\
\text { last year }\end{array}$ \\
\hline $\begin{array}{l}\text { 7. Cyber Dating Abuse Scale in adolescent } \\
\text { dating (ECVPA) } \\
\text { Cava \& Buelga (2018) }\end{array}$ & 4 & $\begin{array}{l}n=363 ; 56.6 \% \text { female } \\
\text { Age }=12-18 \text { years old } \\
\text { Spain }\end{array}$ & $\begin{array}{l}\text { Cyber aggression (10) } \\
(\mathrm{A}, \mathrm{C}) \\
\text { Cyber control (10) (B) }\end{array}$ & $\begin{array}{l}\text { Mobile phone; Social networking sites; Internet; } \\
\text { Whatsapp; Photos; videos and texts }\end{array}$ & $\begin{array}{l}\text { Victimization } \\
\text { perpetration in the } \\
\text { last year }\end{array}$ \\
\hline $\begin{array}{l}\text { 8. Cyber Dating Abuse Questionnaire } \\
\text { (CDAQ-C) } \\
\text { Celsi et al. (2021) }\end{array}$ & 1 & $\begin{array}{l}n=134 \text { heterosexual couples } \\
50 \% \text { female } \\
\text { Age }=18-30 \\
\text { Italy }\end{array}$ & $\begin{array}{c}\text { Items not identified (20) } \\
(\mathrm{A}, \mathrm{B}, \mathrm{C}, \mathrm{E})\end{array}$ & None mentioned & $\begin{array}{l}\text { Victimization } \\
\text { perpetration actual } \\
\text { relationship }\end{array}$ \\
\hline $\begin{array}{l}\text { 9. Experience with Digital Dating Abuse } \\
\text { (EDDA) } \\
\text { Hinduja \& Patchin (2020) }\end{array}$ & 1 & $\begin{array}{l}n=2218 ; 48.1 \% \text { female } \\
\text { Age }=12-17 \text { years old } \\
\text { USA }\end{array}$ & $\begin{array}{l}\text { Dimensions not } \\
\text { identified }(6)(A, B, C)\end{array}$ & $\begin{array}{l}\text { Mobile phone; Tablet; Technological gadgets; } \\
\text { Texts; Online; Photos }\end{array}$ & $\begin{array}{l}\text { Victimization in the last } \\
\text { year }\end{array}$ \\
\hline $\begin{array}{l}\text { 10. Intimate Partner Violence expressed } \\
\text { through Digital Media Scale (EVIME) } \\
\text { Jaen-Cortés et al. (2017) }\end{array}$ & 1 & $\begin{array}{l}n=878 ; 47 \% \text { female } \\
\text { Age }=12-19 \text { years old } \\
\text { Mexico }\end{array}$ & $\begin{array}{l}\text { Control, intrusive } \\
\text { monitoring and } \\
\text { cyber surveillance } \\
(10)(B) \\
\text { Verbal agression (11) } \\
\quad(A) \\
\text { Sexual agression (5) (E) } \\
\text { Sexual coercion (3) (E) } \\
\text { Humiliation (3) }(A, C)\end{array}$ & $\begin{array}{l}\text { Photos; Email; Social networking sites; Texts; } \\
\text { Electronic media; Videos; Mobile phone; GPS; } \\
\text { Phone calls }\end{array}$ & Victimization ever \\
\hline $\begin{array}{l}\text { 11. Cyber Psychological Abuse Scale } \\
\text { (CPAS) } \\
\text { Leisring \& Giumetti (2014) }\end{array}$ & 2 & $\begin{array}{l}n=271 ; 71 \% \text { female } \\
\text { Mean age }=19.03 \text { years } \\
\text { USA }\end{array}$ & $\begin{array}{l}\text { Minor cyber abuse }(6) \\
(A, B, C, D) \\
\text { Severe cyber abuse }(3) \\
(A, B, C)\end{array}$ & $\begin{array}{l}\text { Technological mediums like: Mobile phone; } \\
\text { Email; Computer; Social networking sites }\end{array}$ & $\begin{array}{l}\text { Victimization } \\
\text { perpetration in the } \\
\text { current relationship }\end{array}$ \\
\hline $\begin{array}{l}\text { 12. Digital Intimate Partner Violence } \\
\text { Questionnaire (DIPVQ) } \\
\text { López-Cepero et al. (2018) }\end{array}$ & 1 & $\begin{array}{l}n=449 ; 76 \% \text { female } \\
\text { Mean age }=21.2 \text { years } \\
\text { Lima, Perú }\end{array}$ & $\begin{array}{l}\text { Control centered } \\
\text { cyberabuse (5) (B) } \\
\text { Damage centered } \\
\text { cyberabuse }(7) \\
(\mathrm{A}, \mathrm{C}, \mathrm{E})\end{array}$ & Generic (Internet, Social networks, etc) & $\begin{array}{l}\text { Victimization last } \\
\text { month }\end{array}$ \\
\hline
\end{tabular}


Table 2. Continued.

\begin{tabular}{|c|c|c|c|c|c|}
\hline Instrument/Source & $\begin{array}{l}\text { Num. } \\
\text { study }\end{array}$ & $\begin{array}{l}\text { Sample characteristics (of } \\
\text { the original validation study } \\
\text { and other studies) }\end{array}$ & $\begin{array}{l}\text { Dimensions (Items) } \\
\text { (Cat.) }\end{array}$ & Scope of digital media & $\begin{array}{l}\text { Perspective/Time } \\
\text { frame }\end{array}$ \\
\hline $\begin{array}{l}\text { 13. Cyber Aggression Measure (CAM) } \\
\text { Marganski \& Melander (2015) }\end{array}$ & 2 & $\begin{array}{l}n=804 ; 67 \% \text { female } \\
\text { Age }=18-25 \text { years old } \\
\text { USA }\end{array}$ & $\begin{array}{l}\text { Dimensions not } \\
\text { identified (18) (A,B, } \\
\text { C,E) }\end{array}$ & Socially interactive technologies & $\begin{array}{l}\text { Victimization in the last } \\
\text { year }\end{array}$ \\
\hline $\begin{array}{l}\text { 14. The Cyber Dating Violence Inventory } \\
\text { (TCDVI) } \\
\text { Morelli et al. (2018) }\end{array}$ & 4 & $\begin{array}{l}n=1405 ; 65.1 \% \text { female } \\
\text { Age }=13-22 \text { years old } \\
\text { USA }\end{array}$ & $\begin{array}{l}\text { Psychological violence } \\
(6)(\mathrm{A}, \mathrm{C}) \\
\text { Relational violence }(5) \\
(\mathrm{A}, \mathrm{C})\end{array}$ & Sms; Email; Facebook & $\begin{array}{l}\text { Victimization } \\
\text { perpetration in the } \\
\text { last year }\end{array}$ \\
\hline $\begin{array}{l}\text { 15. Intimate Partner Violence in Social } \\
\text { Media in Adolescents Scale (E-Vpa) } \\
\text { Study 1: Muñiz Rivas \& Monreal } \\
\text { Gimeno (2017) } \\
\text { Study 2: Muñiz Rivas (2017) }\end{array}$ & 1 & $\begin{array}{l}n=919 ; 50.2 \% \text { female } \\
\text { Age }=15-18 \text { years old } \\
\text { Spain } \\
n=919 ; 52,4 \% \text { female } \\
\text { Age }=15-18 \text { years old }\end{array}$ & $\begin{array}{l}\text { Violence (4) (A) } \\
\text { Control (6) (B) }\end{array}$ & Not mentioned & $\begin{array}{l}\text { Perpetration not } \\
\text { specified }\end{array}$ \\
\hline & & Spain & $\begin{array}{l}\text { Emitted Control (6) (B) } \\
\text { Emitted Violence (4) (A) }\end{array}$ & None mentioned & $\begin{array}{l}\text { Perpetration in the last } \\
\text { year }\end{array}$ \\
\hline $\begin{array}{l}\text { 16. Partner Electronic Aggression } \\
\text { Questionnaire (PEAQ) } \\
\text { Preddy (2015) }\end{array}$ & 1 & $\begin{array}{l}\text { Study } 1: n=692 ; 87 \% \text { female } \\
\text { Age }=22 \text { years old; USA } \\
\text { Study 2: } n=513 ; 63 \% \text { female } \\
\text { Age }=19 \text { years old; USA }\end{array}$ & $\begin{array}{l}\text { Public electronic } \\
\text { aggression (4) (C) } \\
\text { Private electronic } \\
\quad \text { aggression (4) (A,B) }\end{array}$ & $\begin{array}{l}\text { Social networking sites; electronic } \\
\text { communication }\end{array}$ & $\begin{array}{l}\text { Victimization } \\
\text { perpetration in the } \\
\text { last } 6 \text { months }\end{array}$ \\
\hline $\begin{array}{l}\text { 17. Digital Dating Abuse Measure } \\
\text { (DDAM-A) } \\
\text { Study 1: Reed et al. (2016) }\end{array}$ & 5 & $\begin{array}{c}n=365 ; 57 \% \text { female; Age }= \\
17-22 \text { years old; USA }\end{array}$ & $\begin{array}{l}\text { Dimensions not } \\
\text { identified (19) (A,B, } \\
\text { C,E) }\end{array}$ & Computer; Internet; Mobile phone; Photo; Video & $\begin{array}{l}\text { Victimization } \\
\text { perpetration } \\
\text { in the last year }\end{array}$ \\
\hline Study 2: Reed et al. (2017) & 5 & $\begin{array}{l}n=696 ; 56 \% \text { female } \\
\text { Age }=13-19 \text { years old; USA }\end{array}$ & $\begin{array}{l}\text { Direct Aggression (8) } \\
\quad(\mathrm{A}, \mathrm{C}) \\
\text { Monitoring/Control } \\
\quad(6)(\mathrm{B}) \\
\text { Sexual Coercion (4) (E) }\end{array}$ & $\begin{array}{l}\text { Mobile phone; Internet; Photo; Video; Message; } \\
\text { Social networking site; Calls; Online accounts }\end{array}$ & $\begin{array}{l}\text { Victimization } \\
\text { perpetration current } \\
\text { or most recent } \\
\text { relationship }\end{array}$ \\
\hline
\end{tabular}


Table 2. Continued.

\begin{tabular}{|c|c|c|c|c|c|}
\hline Instrument/Source & $\begin{array}{l}\text { Num. } \\
\text { study }\end{array}$ & $\begin{array}{l}\text { Sample characteristics (of } \\
\text { the original validation study } \\
\text { and other studies) }\end{array}$ & $\begin{array}{l}\text { Dimensions (Items) } \\
\text { (Cat.) }\end{array}$ & Scope of digital media & $\begin{array}{l}\text { Perspective/Time } \\
\text { frame }\end{array}$ \\
\hline $\begin{array}{l}\text { 18. The Cyber dating Q_A Scale (CQ_AS) } \\
\text { Sánchez et al. (2015) }\end{array}$ & 1 & $\begin{array}{l}n=626 ; 49.6 \% \text { female } \\
\text { Age }=14-17 \text { years old } \\
\text { Spain }\end{array}$ & $\begin{array}{l}\text { Control (6) }(\mathrm{B}) \\
\text { Emotional } \\
\text { communication } \\
\text { strategies }(7)(\mathrm{A}, \mathrm{B}) \\
\text { Jealousy }(4)(\mathrm{B}) \\
\text { Intrusive behaviors } \\
\quad(2)(\mathrm{B}) \\
\text { Cyber dating practices } \\
\text { (4) (D) } \\
\text { Intimacy (3) (NA) }\end{array}$ & $\begin{array}{l}\text { Social networking sites in general; Photos; Texts; } \\
\text { Phone calls; Chat; Mobile phone }\end{array}$ & $\begin{array}{l}\text { Perpetration in the last } 6 \\
\text { months }\end{array}$ \\
\hline $\begin{array}{l}\text { 19. Cyber Dating Abuse Questionnaire } \\
\text { (CDAQ-C) } \\
\text { Smith et al. (2018): Adapted from } \\
\text { cyberbullying scales (Stewart et al., } \\
\text { 2014; Litwiller \& Brausch, 2013) }\end{array}$ & 1 & $\begin{array}{l}n=398 ; 56.4 \% \text { female } \\
\text { Canada } \\
\text { Age }=14-18 \text { years old }\end{array}$ & $\begin{array}{l}\text { Dimensions not } \\
\text { identified (16) (A,B, } \\
\text { C,E) } \\
\text { - Victimization (8) } \\
\text { - Perpetration (8) }\end{array}$ & Online; Photos; Videos & $\begin{array}{l}\text { Victimization } \\
\text { perpetration in the } \\
\text { last year }\end{array}$ \\
\hline $\begin{array}{l}\text { 20. Electronic Dating Aggression Survey } \\
\text { (EDAS) } \\
\text { Smith-Darden et al. (2017) adapted } \\
\text { from Finkelor et al. (2000) }\end{array}$ & 1 & $\begin{array}{l}n=727 ; 51 \% \text { female } \\
\text { Age }=11-15 \text { years old } \\
\text { USA }\end{array}$ & $\begin{array}{l}\text { Dimensions identified, } \\
\text { but items not } \\
\text { specified (12) } \\
\text { - Cyberstalking (B) } \\
\text { - Harassment (C) } \\
\text { - Coercive sexting (E) }\end{array}$ & Not mentioned & $\begin{array}{l}\text { Perpetration in the last } \\
\text { year }\end{array}$ \\
\hline $\begin{array}{l}\text { 21. Technology Assisted Adolescent } \\
\text { Violence (TAAV) } \\
\text { Stonard (2018) }\end{array}$ & 2 & $\begin{array}{l}n=469 ; 52 \% \text { female } \\
\text { Age: } 12-18 \text { years old } \\
\text { England }\end{array}$ & $\begin{array}{l}\text { Dimensions } \\
\text { identified, but } \\
\text { items not specified } \\
(12)(\mathrm{A}, \mathrm{B}, \mathrm{C}, \mathrm{E})\end{array}$ & Not mentioned & $\begin{array}{l}\text { Victimization } \\
\text { perpetration last } 12 \\
\text { months }\end{array}$ \\
\hline $\begin{array}{l}\text { 22. Electronic Dating Aggression (EDA) } \\
\text { Thulin et al. (2020) }\end{array}$ & 1 & $\begin{array}{l}n=470 \\
62.5 \% \text { female } \\
\text { Age }=11-16 \\
\text { USA }\end{array}$ & $\begin{array}{l}\text { Monitoring (2) (B) } \\
\text { Harassment }(6)(C) \\
\text { Sexual coercion (3) (E) }\end{array}$ & $\begin{array}{l}\text { Text; Email; Social network Voicemail; Cell } \\
\text { Phone; Calls; IM; Chat; Pictures; Videos }\end{array}$ & $\begin{array}{l}\text { Victimization } \\
\text { Perpetration not } \\
\text { mentioned }\end{array}$ \\
\hline
\end{tabular}


Table 2. Continued.

\begin{tabular}{|c|c|c|c|c|c|}
\hline Instrument/Source & $\begin{array}{l}\text { Num. } \\
\text { study }\end{array}$ & $\begin{array}{l}\text { Sample characteristics (of } \\
\text { the original validation study } \\
\text { and other studies) }\end{array}$ & $\begin{array}{l}\text { Dimensions (Items) } \\
\text { (Cat.) }\end{array}$ & Scope of digital media & $\begin{array}{l}\text { Perspective/Time } \\
\text { frame }\end{array}$ \\
\hline $\begin{array}{l}\text { 23. Partner Directed Cyber Aggression } \\
\text { Scale (PDCAS) } \\
\text { Wright (2015), adapted from Linder } \\
\text { et al. (2002) }\end{array}$ & 1 & $\begin{array}{l}n=600 ; 54 \% \text { female } \\
\text { Age }=17-18 \text { years old } \\
\text { USA }\end{array}$ & $\begin{array}{l}\text { Relational aggression } \\
\text { (3) (A) } \\
\text { Privacy invasion (2) (B) }\end{array}$ & Online; Texts & $\begin{array}{l}\text { Perpetration in the last } \\
\text { year }\end{array}$ \\
\hline $\begin{array}{l}\text { 24. Cyber Dating Abuse Questionnaire } \\
\text { (CDAQ-D) } \\
\text { Zweig et al. (2013) adapted from Picard } \\
\text { (2007) }\end{array}$ & 8 & $\begin{array}{l}n=5647 ; 52 \% \text { female } \\
\text { Age }=12-18 \text { years old } \\
\text { USA }\end{array}$ & $\begin{array}{l}\text { Sexual abuse (4) (E) } \\
\text { Non-sexual } \\
\text { abuse (12) (E) }\end{array}$ & $\begin{array}{l}\text { Photos; Texts; Email; Instant messaging services; } \\
\text { Chat rooms; Social networking sites; Video; } \\
\text { Mobile phone }\end{array}$ & $\begin{array}{l}\text { Victimization } \\
\text { perpetration in the } \\
\text { last year }\end{array}$ \\
\hline
\end{tabular}

Note. Cat. = Categorization $(\mathrm{A}=$ Cyber psychological aggression; $\mathrm{B}=$ Cyber control $; \mathrm{C}=$ Public harassment; $\mathrm{D}=$ Exclusion; $\mathrm{E}=\mathrm{Cyber}$ sexual aggression $)$; $\mathrm{NA}=$ non-applied. 
Table 3. Summary of the Quality Assessment of the CDV Measurement Instruments based on the COSMIN Guide

\begin{tabular}{|c|c|c|c|c|c|c|c|c|c|c|}
\hline \multirow[b]{2}{*}{ Instrument } & \multicolumn{2}{|c|}{ Content Validity } & \multicolumn{2}{|c|}{ Structural Validity } & \multicolumn{2}{|c|}{ Internal Consistency } & \multicolumn{2}{|c|}{ Construct Validity } & \multicolumn{2}{|c|}{ Recommendation } \\
\hline & MQ & Result (OR) & MQ & Result (OR) & $\mathrm{MQ}$ & Result (OR) & MQ & Result (OR) & TSR/QE & Cat \\
\hline 1. EV & MOD & DS: +; RR: + & & & VG & $\alpha=.73-.77(+)$ & VG & $2 / 3(+)$ & $3 / \mathrm{H}$ & A \\
\hline 2. CDAQ-A & $\mathrm{VL}$ & RR:? & & & & & IN & No Hyp. (?) & $\mathrm{VL}$ & $\mathrm{C}$ \\
\hline 3. CDAQ-B & MOD & & & & & & & & & \\
\hline Study 1 & & DS: +; RR: + & VG & $\mathrm{CFI}=.99(+)$ & VG & $\alpha=.73-.84(+)$ & VG & $2 / 2(+)$ & $5 / \mathrm{H}$ & $\mathbf{A}$ \\
\hline Study 2 & & DS: +; RR: + & VG & $\mathrm{NNFI}=.96(+)$ & VG & $\alpha=.84-.91(+)$ & & & $4 / \mathrm{H}$ & \\
\hline Study 3 & & DS: +; RR: + & VG & $\mathrm{CFI}=.97(+)$ & IN & $\alpha=.97(+)$ & & & $3 / \mathrm{M}$ & \\
\hline Study 4 & & DS: +; RR: + & VG & $\mathrm{CFI}=.938(?)$ & VG & $\alpha=.79-.89(+)$ & VG & $1 / 1(+)$ & $4 / \mathrm{H}$ & \\
\hline 4. TAR & $\mathrm{HI}$ & $\begin{array}{l}\text { DS: +; CVS: +; } \\
\text { RR: }+\end{array}$ & $\mathrm{AD}$ & $\mathrm{KMO}=.858-.946(+)$ & VG & $\alpha=.80-.88(+)$ & VG & $2 / 3(+)$ & $6 / \mathrm{H}$ & $\mathbf{A}$ \\
\hline 5. CPI & VL & RR:? & & & IN & $\alpha=.90(+)$ & & & VL & $\mathrm{C}$ \\
\hline 6. CARPA & MOD & DS: +; RR: + & VG & $\mathrm{CFI}=1(+)$ & VG & $\alpha=.75-.97(+)$ & VG & $1 / 1(+)$ & $5 / \mathrm{H}$ & $\mathbf{A}$ \\
\hline 7. ECVPA & MOD & DS: +; RR: ? & VG & $\mathrm{CFI}=.99(+)$ & VG & $\alpha=.92-.97(+)$ & VG & No Hyp. (?) & $3 / \mathrm{H}$ & B \\
\hline 8. CDAQ-C & VL & RR: + & VG & $\mathrm{CFI}=.99(+)$ & VG & $\alpha=.91-.95(+)$ & VG & $3 / 3(+)$ & $4 / \mathrm{M}$ & A \\
\hline 9. EDDA & VL & RR:? & & & DB & $\alpha=.85(+)$ & VG & No Hyp. (?) & $1 / \mathrm{VL}$ & $\mathrm{C}$ \\
\hline 10. EVIME & MOD & DS: +; RR: ? & $\mathrm{AD}$ & $\mathrm{KMO}=.936(+)$ & VG & $\alpha=.78-.93(+)$ & & & $3 / \mathrm{M}$ & B \\
\hline 11. CPAS & MOD & DS: +; RR: ? & VG & $\mathrm{CFI}=.97(+)$ & IN & $\alpha=.81-.82(+)$ & VG & No Hyp. (?) & $2 / \mathrm{M}$ & B \\
\hline 12. DIPVQ & MOD & DS: +; RR: + & $\mathrm{AD}$ & $\mathrm{KMO}=.93(+)$ & VG & $\alpha=.96-.97(+)$ & & & $4 / \mathrm{M}$ & $\mathrm{A}$ \\
\hline 13. CAM & VL & $\mathrm{RR}:+$ & & & VG & $\alpha=.50-.92(?)$ & VG & $2 / 2(+)$ & $2 / \mathrm{L}$ & $\mathrm{C}$ \\
\hline 14. TCDVI & $\mathrm{VL}$ & RR: - & VG & $\mathrm{CFI}=.97(+)$ & VG & $\alpha=.81-.82(+)$ & VG & No Hyp. (?) & $2 / \mathrm{M}$ & C \\
\hline \multicolumn{11}{|l|}{ 15. E-VPA } \\
\hline Study 1 & VL & RR: - & & & VG & $\alpha=.80-.86(+)$ & VG & No Hyp. (?) & $1 / \mathrm{M}$ & $\mathrm{C}$ \\
\hline Study 2 & & RR: - & VG & $\mathrm{CFI}=.91(?)$ & VG & $\alpha=.80-.86(+)$ & VG & No Hyp. (?) & $1 / \mathrm{M}$ & $\mathrm{C}$ \\
\hline 16. PEAQ & MOD & DS: +; RR: ? & $\mathrm{AD}$ & $\mathrm{KMO}=.94(+)$ & VG & $\alpha=.93-.97(+)$ & & & $3 / \mathrm{M}$ & \\
\hline 17. DDAM-A & VL & & & & & & & & & \\
\hline Study 1 & & RR: + & & & VG & $\alpha=.73-.77(+)$ & VG & No Hyp. (?) & $2 / \mathrm{M}$ & B \\
\hline Study 2 & & RR: + & & & VG & $\alpha=.67-.83(+)$ & VG & $3 / 4(+)$ & $3 / \mathrm{M}$ & B \\
\hline 18. CQ_AS & MOD & DS: +; RR: ? & VG & $\mathrm{CFI}=.97(+)$ & VG & $\alpha=.71-.85(+)$ & & & $3 / \mathrm{H}$ & B \\
\hline 19. CDAQ-D & $\mathrm{VL}$ & RR: + & & & IN & $H=.86-.86(+)$ & VG & No Hyp. (?) & $2 / \mathrm{L}$ & $\mathrm{C}$ \\
\hline 20. EDAS & VL & RR: ? & & & VG & $\alpha=.47-.77(?)$ & VG & $1,5 / 2(+)$ & $1 / \mathrm{L}$ & $\mathrm{C}$ \\
\hline 21. TAAV & MOD & DS: +; RR: + & & & VG & $\alpha=.86-.99(+)$ & VG & $2 / 3(+)$ & $4 / \mathrm{M}$ & A \\
\hline 22. EDA & $\mathrm{VL}$ & RR: ? & VG & RMSEA $=.017(+)$ & IN & $\alpha=.80-.83(+)$ & VG & No Hyp. (?) & $2 / \mathrm{L}$ & C \\
\hline 23. PDCAS & VL & RR: - & VG & $\mathrm{CFI}=.94(?)$ & VG & $\alpha=.82-.91(+)$ & VG & $3 / 3(+)$ & $2 / \mathrm{L}$ & $\mathrm{C}$ \\
\hline 24. CDAQ-E & VL & RR: - & & & VG & $\alpha=.81-.92(+)$ & VG & $3 / 4(+)$ & $2 / \mathrm{L}$ & $\mathrm{C}$ \\
\hline
\end{tabular}

Note. COSMIN Risk of Bias Checklist rating. $\mathrm{MQ}=$ Methodological quality $(\mathrm{VG}=$ Very Good; $\mathrm{HI}=$ High; $\mathrm{AD}=\mathrm{Adequate} ; \mathrm{MOD}=\mathrm{Moderate} ; \mathrm{DB}=\mathrm{Doubtful} ; \mathrm{IN}=\mathrm{Inadequate} ; \mathrm{VL}=\mathrm{Very}$ Low); OR = overall rating; blank cells indicate no results. Result of measurement property = + = Sufficient; $+/-=$ Inconsistent; $-=$ Insufficient; ? = Indeterminate. DS = Development Study; $\mathrm{RR}=$ Reviewers Rating; CVS = Content Validity Study; No Hyp. = No Hypothesis; TSR = Total sufficient rating; QE: Quality of evidence; Cat. = Categories for recommendations on suitable instruments ( $\mathrm{A}$ = best suitable; $\mathrm{A}=$ recommended; $\mathrm{B}=$ with caution; $\mathrm{C}=$ not recommended). 
unidimensional, leading to uncertainty regarding whether internal consistency should apply (Mokkink et al., 2018). The remaining 22 studies that assessed internal consistency received a "very good" rating as it was calculated for each subscale and adhered to the other requisites for assessing this property.

\section{Hypotheses Testing for Construct Validity}

This property refers to the degree to which the results of an instrument are consistent with the hypotheses, assuming that it validly measures the construct to be measured (Mokkink et al., 2018). Many types of hypotheses can be tested but, in general, they concern comparisons with other outcome measurement instruments. In fact, every study that gave information about this property made comparisons with other outcome measures. However, ten of the studies that made these comparisons did not formulate specific hypotheses, leading to indeterminate results for this property.

\section{Best Instruments for Assessing CDV}

The current systematic review has detected the following three instruments as the most suitable for the assessment of CDV for adolescents and young people in the population of interest, based on their psychometric properties:

Cyber Dating Abuse Questionnaire (CDAQ-B; Borrajo, Gámez-Guadix, Pereda, et al., 2015). Four psychometric studies were identified (Borrajo, Gámez-Guadix, Pereda, et al., 2015; Caridade \& Braga, 2019; HidalgoRasmussen et al., 2020; Lara, 2020) reporting on the psychometric properties of the CDAQ-B (Borrajo, Gámez-Guadix, Pereda, et al., 2015) based on adolescents and young adults. This instrument was adapted to four cultural contexts (Spain, Portugal, Chile and Mexico). The adaption studies of this instrument (Caridade \& Braga, 2019; Hidalgo-Rassmusen et al., 2020; Lara, 2020) were carried out appropriately by consulting with experts, focus groups or involving the target population. These studies modified some of the items due to wording and context, and two of them used the instrument with a sample of adolescents (13-24 years), despite the original (Borrajo, Gámez-Guadix, Pereda, et al., 2015) having been used in a sample of young adults (18-30 years). The three adaption studies showed positive and good-quality evidence in their psychometric properties, which means that this instrument is suitable for other contexts, with few changes. Moreover, this instrument has been more widely applied (23 studies) in comparison with other instruments (varying from 1 to 8 studies). The original study showed positive evidence in four indicators (content validity, structural validity, internal consistency, and construct validity). There was moderate-quality evidence supporting content validity. This instrument included 20 items related to three dimensions of CDV (Cyber psychological aggression, Cyber control, and Public harassment), but Cyber sexual and Exclusion were not included. This instrument could be highly recommended for assessing CDV in young people, but its application in adolescents under 14 years of age may be doubtful because the four psychometric studies did not cover the age range of adolescence, for which more validation studies are needed.

Technology-facilitated Abuse in Relationships Scale (TAR; Brown \& Hegarty, 2021). A psychometric study of TAR (Brown \& Hegarty, 2021) was carried out with 30 items in a population of 16-24 year-olds. High-quality evidence supporting content validity was found, and one content validity study was also rated as sufficient. The quality of the evidence for structural validity was adequate, with one study reporting a four-factor structure via Exploratory Factor Analysis. This instrument provided a clear definition of the dating relationship, the period of time under consideration is well delimited, and measures behaviors on all digital devices and digital platforms. The items are related to four dimensions of CDV (Cyber psychological aggression, Cyber control, Public harassment, and Cyber sexual). This instrument is very promising for assessing CDV abuse in old adolescents and young adults, although it should be noted that it was applied in Australia only. Thus, its application should not be recommended in other languages or countries.

Abuse in Teen Relationships (CARPA; Calvete et al., 2021). One psychometric study examined the measurement properties of the CARPA (Calvete et al., 2021) based on a sample of Spanish adolescents (11-18 years). Moderate-quality evidence supporting content validity was found. The quality of the evidence of structural validity was very good, with a two-factor structure applying Confirmatory Factor Analysis. This instrument is a subscale inside a more complete instrument assessing all kinds of abuse in dating relationships, so it is more reduced than the other two instruments. It included 11 items related to four dimensions of CDV (Cyber psychological aggression, Cyber control, Public harassment, and Cyber sexual). This instrument showed good measurement properties and it is promising, but it has only been applied in Spanish samples, so adaption studies should be done to prove its suitability for other contexts.

\section{Discussion}

CDV is a relatively new line of research, and there is no consensus regarding its conceptualization, a situation which has led to the development of multiple and different tools to measure it. The assessment of CDV shows 
a great methodological diversity (Rodríguez-Dominguez et al., 2020), and the objective of this study was to realize a systematic review of the CDV literature, describe the measurement tools that have been developed in recent years and, finally, identify the best instruments based on their methodological quality. It is hoped this paper will help scholars in choosing an appropriate instrument for their research.

In this systematic review, up to 24 different measurement tools were found, along with 29 psychometric studies about these instruments, all presenting differences in methodological characteristics such as sampling context, psychometric properties provided, and dimensions studied. This variability in the measurement tools is not new as it had already been seen in previous reviews (Brown \& Hegarty, 2018; Caridade et al., 2019; Cavalcanti \& Coutinho 2019; RodríguezDomínguez et al., 2020). However, this paper includes some new instruments developed in recent years, and provides a deep evaluation of the psychometric properties of the measurement tools and its methodological quality following the COSMIN guidelines (Table 3) in order to formulate recommendations regarding their use. Despite the numerous instruments and psychometric studies found by this review, many development and content validity studies did not report a systematic process of how items were produced or selected, did not involve target population or experts, or did not provide sufficient detail (such as items, recall period, and response options).

After the evaluation of ten indicators of methodological quality as well as the eight indicators assessing the reported psychometric properties quality, seven selected instruments were classified as category A (Prinsen et al., 2018), and three instruments were determined as the most suitable for the measurement of CDV: Cyber Dating Abuse Questionnaire (Borrajo, GámezGuadix, Pereda, et al., 2015), Technology-facilitated Abuse in Relationships Scale (Brown \& Hegarty, 2021), and Abuse in Teen Relationships (CARPA) (Calvete et al., 2021). The Cyber Dating Abuse Questionnaire could be considered as the best instrument because it shows good-quality evidence in its psychometric properties, with consistent evidence of four psychometric studies based on adolescents and young adults and adapted to four cultural contexts (Spain, Portugal, Chile and Mexico). Thus, this instrument is recommended for assessing CDV in adolescents older than 14 and young adults. The Technology-facilitated Abuse in Relationships Scale stands out for having highquality evidence supporting content validity. Moreover, it measures CDV behaviors on all digital devices and includes four dimensions. However, it was only applied to an Australian context. The Abuse in Teen Relationships scale has a cyber subscale with eleven items and can be applied to adolescents of all ages, but adaption studies should be done to prove its suitability for non-Spanish contexts. Nevertheless, no selected instrument assessed or provided psychometric evidence to support cross-cultural validity, reliability, criterion validity, or responsiveness.

The search in the present study was limited by focusing only on scientific articles, by excluding measures that investigate only one CDV behavior and those studies focused on subjects older than 30 years, which may have resulted in relevant contributions to the review being overlooked. COSMIN is a rigorous methodological tool, but there is a certain level of subjectivity in rating each paper (Cassidy et al., 2018). Thus, the process of evaluation of psychometric properties of 24 instruments is transparent, and the global rating of instruments was elaborated by consensus between two evaluators considering the information in Table 3.

Digital media such as cell phones and social networks have had a favorable impact on romantic relationships, since couples can keep in touch when they are not face to face, allowing the perception of greater closeness (Javier-Juárez et al., 2021). However, digital media can also have a negative impact in dating relationships because they provide new channels to exercise harassment, control and abuse (and therefore to suffer them), in childhood, adolescence and young adulthood (van Ouytsel et al., 2019). There is no agreement regarding the conceptualization of CDV. This paper defends the use of the term cyber dating violence, and the definition includes different types of dating aggression using digital media (psychological direct, control, public harassment, exclusion, and sexual). Exclusion (excluding, or blocking on social networks) is the least assessed dimension among the instruments analyzed. Although previous reviews (Brown \& Hegarty, 2018; Caridade et al., 2019; Cavalcanti \& Coutinho, 2019; Rodríguez-Domínguez et al., 2020) have already shown the variability of the used instruments and their methodological approaches, the present review analyzed the quality of psychometric properties using the COSMIN checklist. Thus, this systematic review can help researchers to assess CDV and clinicians to focus on the evaluation programs. Among the three tools recommended, the Cyber Dating Abuse Questionnaire (14-30 years) obtained the highest total sufficient rating.

Some studies argue that, although online and offline violence present some similarities in terms of habits and attitudes, violence through virtual contexts shows certain particular characteristics that should be addressed in a specific way (Núñez et al., 2021). Thus, it is important to understand the negative use of technology in intimate relationships, as well as the differences and similarities between online and offline violence, in order to be able to support adolescents and young people 
during this important stage of their lives, and to advance in the creation of effective and specific prevention and treatment programs. Early detection of CDV could be very relevant; therefore, it is essential to provide a reliable and valid evaluation instrument. Although the communication is virtual, the relationships, their effects and consequences are real. What happens online is not something merely virtual since it has real effects on people and can cause more depressive moods (Cava, Buelga et al., 2020) and a significant deterioration of life quality. Future studies could focus on analyzing reliability, criterion and cross-cultural validity of the existing instruments.

\section{References}

The psychometric studies analyzed in the paper and those included in Appendix A have an asterisk $\left({ }^{*}\right)$.

*Araci-İyiaydin, A., Toplu-Demirtaş, E., Akçabozan-

Kayabol, N. B., \& Fincham, F. D. (2020). I ruminate therefore I violate: The tainted love of anxiously attached and jealous partners. Journal of Interpersonal Violence. Advance online publication. https: / / doi.org/10.1177/0886260520967743

*Bennett, D. C., Guran, E. L., Ramos, M. C., \& Margolin, G. (2011). College students' electronic victimization in friendships and dating. Violence and Victims, 26(4), 410-429. https:/ / doi.org/10.1891/0886-6708.26.4.410

*Bhogal, M. S., \& Howman, J. M. (2019). Mate value discrepancy and attachment anxiety predict the perpetration of digital dating abuse. Evolutionary Psychological Science, 5, 113-120. https: / / doi.org/10.1007/s40806-018-0172-6

*Bhogal, M. S., Rhead, C., \& Tudor, C. (2019). Understanding digital dating abuse from an evolutionary perspective: Further evidence for the role of mate value discrepancy. Personality and Individual Differences, 151, Article 109552. https://doi.org/10.1016/j.paid.2019.109552

*Bhogal, M. S., Tudor, C., \& Hira, S. (2021). The role of matingrelevant factors in the perpetration of digital dating abuse. Journal of Interpersonal Violence. Advance online publication. https: / / doi.org/10.1177/08862605211004103

*Borrajo, E., \& Gámez-Guadix, M. (2016). Cyber dating abuse: Its link to depression, anxiety and dyadic adjustment. Behavioral Psychology, 24(2), 221-235.

*Borrajo, E., Gámez-Guadix, M., \& Calvete, E. (2015a). Justification beliefs of violence myths about and cyber dating abuse. Psicothema, 27(4), 327-333. https:/ / doi.org/10.7334/ psicothema2015.59

*Borrajo, E., Gámez-Guadix, M., \& Calvete, E. (2015b). Cyber dating abuse: Prevalence, context, and relationship with offline dating aggression. Psychological Reports, 116(2), 565-585. http:/ / doi.org/10.2466/21.16.PR0.116k22w4

*Borrajo, E., Gámez-Guadix, M., Pereda N., \& Calvete, E. (2015). The development and validation of the cyber dating abuse questionnaire among young couples. Computers in Human Behavior, 48, 358-365. https:/ / doi.org/10.1016/ j.chb.2015.01.063

*Brem, M. J., Romero, G., Garner, A. R., Grigorian, H., \& Stuart, G. L. (2019). Alcohol problems, jealousy, and cyber dating abuse perpetration among men and women: Toward a conceptual model. Journal of Interpersonal Violence, 36,
NP10205-NP10228. https: / / doi.org/10.1177/ 0886260519873333

*Brem, M. J., Stuart, G. L., Cornelius, T. L., \& Shorey, R. C. (2019). A longitudinal examination of alcohol problems and cyber, psychological, and physical dating abuse: The moderating role of emotion dysregulation. Journal of Interpersonal Violence, 36, NP10499-NP10519. https:/ / doi.org/10.1177/0886260519876029

Brown, C., \& Hegarty, K. (2018). Digital dating abuse measures: A critical review. Aggression and Violent Behavior, 40, 44-59. https: / / doi.org/10.1016/j.avb.2018.03.003

*Brown, C., \& Hegarty, K. (2021). Development and validation of the TAR Scale: A measure of technology-facilitated abuse in relationships. Computers in Human Behavior Reports, 3, Article 100059. https: / / doi.org/10.1016/j.chbr.2021.100059

*Brown, C., Sanci, L., \& Hegarty, K. (2021). Technologyfacilitated abuse in relationships: Victimisation patterns and impact in young people. Computers in Human Behavior, 124, Article 106897. https:/ / doi.org/10.1016/j.chb.2021.106897

${ }^{*}$ Burke, S. C., Wallen, M., Vail-Smith, K., \& Knox, D. (2011). Using technology to control intimate partners: An exploratory study of college undergraduates. Computers in Human Behavior, 27, 1162-1167. https:/ / doi.org/10.1016/ j.chb.2010.12.010

*Calvete, E., Fernández-González, L., Orue, I., Machimbarrena, M., \& González-Cabrera, J. (2021). Validación de un cuestionario para evaluar el abuso en relaciones de pareja en adolescentes (CARPA), sus razones y las reacciones [Validation of a questionnaire to assess abuse in dating relationships in Spanish adolescents (CARPA):

Reasons and reactions]. Revista de Psicología Clínica con Niños y Adolescentes, 8(1), 60-69. http:/ / doi.org/10.21134/ rpcna.2021.08.1.8

${ }^{*}$ Caridade, S. M., \& Braga, T. (2019). Versão portuguesa do Cyber Dating Abuse Questionaire (CDAQ) - Questionário sobre Ciberabuso no Namoro (CibAN): Adaptação e propriedades psicométricas [The Portuguese version of the Cyber Dating Abuse Questionnaire (CDAQ): Adapting and psychometric properties]. Análise Psicológica, 37, 93-105.

http:/ / doi.org/105.10.14417/ap.1543

Caridade, S., Braga, T., \& Borrajo, E. (2019). Cyber dating abuse (CDA): Evidence from a systematic review. Aggression and Violent Behavior, 48, 152-168. https: / / doi.org/10.1016/ j.avb.2019.08.018

*Caridade, S., Sousa, H. F. P., \& Dinis, M. A. P. (2020). Cyber and offline dating abuse in a Portuguese sample: Prevalence and context of abuse. Behavioral Sciences, 10, Article 152. https: / / doi.org/10.3390/bs10100152

Cassidy, S. A., Bradley, L., Bowen, E., Wigham, S., \& Rodgers, J. (2018). Measurement properties of tools used to assess suicidality in autistic and general population adults: A systematic review. Clinical Psychology Review, 62, 56-70. http:/ / doi.org/10.1016/j.cpr.2018.05.002

*Cava, M.-J., \& Buelga, S. (2018). Propiedades psicométricas de la Escala de Ciber-Violencia en Parejas Adolescentes (CibVPA) [Psychometric properties of the Cyber-Violence Scale in Adolescent Couples (Cib-VPA)]. Suma Psicológica, 25, 51-61. http:/ / doi.org/10.14349/sumapsi.2018.v25.n1.6

*Cava, M.-J., Buelga, S., Carrascosa, L., \& Ortega-Barón, J. (2020). Relations among romantic myths, offline dating violence victimization and cyber dating violence 
victimization in adolescents. International Journal of Environmental Research and Public Health, 17, Article 1551. http:/ / doi.org/10.3390/ijerph17051551

*Cava, M.-J., Martínez-Ferrer, B., Buelga, S., \& Carrascosa, L. (2020). Sexist attitudes, romantic myths, and offline dating violence as predictors of cyber dating violence perpetration in adolescents. Computers in Human Behavior, 111, Article 106449. https:/ / doi.org/10.1016/ j.chb.2020.106449

*Cava, M.-J., Tomás, I., Buelga, S., \& Carrascosa, L. (2020). Loneliness, depressive mood and cyberbullying victimization in adolescent victims of cyber dating violence. International Journal of Environmental Research and Public Health, 17(12), Article 4269. https: / / doi.org/10.3390 / ijerph17124269

*Celsi, L., Paleari, F. G., \& Fincham, F. D. (2021). Adverse childhood experiences and early maladaptive schemas as predictors of cyber dating abuse: An actor-partner interdependence mediation model approach. Frontiers in Psychology, 12, Article 623646. https:/ / doi.org/10.3389/ fpsyg. 2021.623646

Cavalcanti, J. G., \& Coutinho, M. d. P. d. L. (2019). Abuso digital en las relaciones amorosas: Una revisión sobre prevalencia, instrumentos de evaluación y factores de riesgo [Cyber dating abuse: A review of prevalence, evaluation instruments and risk factors]. Avances En Psicología Latinoamericana, 37(2), 235-254. https: / / doi.org/10.12804/ revistas.urosario.edu.co/apl/a.6888

*Dank, M., Lachman, P., Zweig, J. M., \& Yahner, J. (2013). Dating violence experiences of lesbian, gay, bisexual, and transgender youth. Journal of Youth and Adolescence, 43, 846-857. https: / / doi.org/10.1007/s10964-013-9975-8

*Deans, H., \& Bhogal, M. S. (2019). Perpetrating cyber dating abuse: A brief report on the role of aggression, romantic jealousy and gender. Current Psychology 38, 1077-1082. https: / / doi.org/10.1007/s12144-017-9715-4

*Dyar, C., Feinstein, B. A., Zimmerman, A. R., Newcomb, M. E., Mustanski, B., \& Whitton, S. W. (2020). Dimensions of sexual orientation and rates of intimate partner violence among young sexual minority individuals assigned female at birth: The role of perceived partner jealousy. Psychology of Violence, 10(4), 411-421. https: / / doi.org/10.1037/ vio0000275

Duerksen, K. N., \& Woodin, E. M. (2019). Technological intimate partner violence: Exploring technology-related perpetration factors and overlap with in-person intimate partner violence. Computers in Human Behavior, 98, 223-231. https: / / doi.org/10.1016/j.chb.2019.05.001

*Ellyson, A. M., Adhia, A., Lyons, V. H., \& Rivara, F. P. (2021). Prevalence, age of initiation, and patterns of co-occurrence of digital dating abuse behaviors nationwide. Children and Youth Services Review, 122, Article 105921. https:/ / doi.org/ 10.1016/j.childyouth.2020.105921

*Fernández-González, L., Calvete, E., \& Sánchez-Álvarez, N. (2020). Efficacy of a brief intervention based on an Incremental Theory of Personality in the prevention of adolescent dating violence: A randomized controlled trial. Psychosocial Intervention, 29(1), 9-18. https: / / doi.org/ 10.5093/pi2019a14
Fernet, M., Lapierre, A., Hébert, M., \& Cousineau, M.-M. (2019). A systematic review of literature on cyber intimate partner victimization in adolescent girls and women. Computers in Human Behavior, 100, 11-25. https:/ / doi.org/ 10.1016/j.chb.2019.06.005

*Finkelhor, D., Mitchell, K. J., \& Wolak, J. (2000). Online victimization: A report on the nation's youth. National Center for Missing and Exploited Children, Alexandria, VA.; Crimes against Children Research Center, Durham, NH.

${ }^{*}$ Gracia-Leiva, M., Puente-Martínez, A., Ubillos-Landa, S., González-Castro, J. L., \& Páez-Rovira, D. (2020). Off- and online heterosexual dating violence, perceived attachment to parents and peers and suicide risk in young women. International Journal of Environmental Research and Public Health, 17(9), Article 3174. http:/ / doi.org/10.3390/ ijerph17093174

*Hidalgo-Rasmussen, C. A., Javier-Juárez, P., Zurita-Aguilar, K., Yáñez-Peñuñuri, L., Franco-Paredes, K., \& ChávezFlores, V. (2020). Adaptación transcultural del "Cuestionario de Abuso Cibernético en la Pareja" (CDAQ) para adolescentes mexicanos. [Cross-cultural adaptation of the "Cyber Dating Abuse Questionnaire" (CDAQ) in Mexican adolescents]. Behavioral Psychology/Psicología Conductual, 28 (3), 435-453.

${ }^{*}$ Hinduja, S., \& Patchin, J. W. (2020). Digital dating abuse among a national sample of U.S. youth. Journal of Interpersonal Violence, 36, 11088-11108. https: / / doi.org/ $10.1177 \% 2 \mathrm{~F} 0886260519897344$

Ibabe, I., Arnoso, A., \& Elgorriaga, E. (2020). Child-to-parent violence as an intervening variable in the relationship between inter-parental violence exposure and dating violence. International Journal of Environmental Research and Public Health, 17(5), 1514. http:/ / dx.doi.org/10.3390/ ijerph17051514

*Jaen-Cortés, C. I., Rivera-Aragón, S., Reidl-Martínez, L. M., \& García-Méndez, M. (2017). Violencia de pareja a través de medios electrónicos en adolescentes mexicanos [Intimate partner violence through electronic media in Mexican adolescents]. Acta de Investigación Psicológica, 7, 2593-2605. http:/ / doi.org/10.1016/ j.aipprr.2017.01.001

*Javier-Juárez, S. P., Hidalgo-Rasmussen, C. A., DíazReséndiz, F. d. J., \& Vizcarra-Larrañaga, M. B. (2021). Abuso cibernético en el noviazgo y relación intrafamiliar en adolescentes estudiantes mexicanos. [Cyber abuse in dating and intrafamily relationships in Mexican teenage students]. Behavioral Psychology/Psicología Conductual, 29(1), 127-143. https: / / doi.org/10.51668/bp.8321107s

${ }^{*}$ Lara, L. (2020). Cyber dating abuse: Assessment, prevalence, and relationship with offline violence in young Chileans. Journal of Social and Personal Relationships, 37(5), 1681-1699. https:/ /doi.org/10.1177/0265407520907159

*Leisring, P. A., \& Giumetti, G. W. (2014). Sticks and stones may break my bones, but abusive text messages also hurt: Development and validation of the Cyber Psychological Abuse Scale. Partner Abuse, 5(3). http:/ / doi.org/10.1891/ 1946-6560.5.3.323

${ }^{*}$ Linder, J. R., Crick, N. R., \& Collins, W. A. (2002). Relational aggression and victimization in young adults' romantic 
relation- ships: Associations with perceptions of parent, peer, and romantic relationship quality. Social Development, 11(1), 69-86. https: / / doi.org/10.1111/1467-9507.00187

*Litwiller, B. J., \& Brausch, A. M., (2013). Cyber bullying and physical bullying in adolescent suicide: the role of violent behavior and substance use. Journal of Youth and Adolescene. 42, 675-684. https: / / doi.org/10.1007/ s10964-013-9925-5

*López-Cepero, J., Vallejos-Saldarriaga, J., \& Merino-García, M. (2018). Digital intimate partner violence among Peruvian youths: Validation of an instrument and a theoretical proposal. Journal of Interpersonal Violence, 36(11-12), 5167-5185. https: / / doi.org/10.1177/0886260518803610

${ }^{*}$ Lu, Y., Shin, Y., Le, V. D., Temple, J. R., \& Pettigrew, J. (2020). Prevalence of teen dating violence and the associations with substance use and externalizing behaviors in Nicaraguan early adolescents. Health Education, 120(2), 165-177. https: / / doi.org/10.1108/HE-01-2020-0006

${ }^{*}$ Lu, Y., van Ouytsel, J., Walrave, M., Ponnet, K., \& Temple J. R. (2018). Cross-sectional and temporal associations between cyber dating abuse victimization and mental health and substance use outcomes. Journal of Adolescence, 65, 1-5. https:/ / doi.org/10.1016/j.adolescence.2018.02.009

*Machimbarrena, J. M., Calvete, E., Fernández-González, L., Álvarez-Bardón, A., Álvarez-Fernández, L., \& GonzálezCabrera, J. (2018). Internet risks: An overview of victimization in cyberbullying, cyber dating abuse, sexting, online grooming and problematic internet use. International Journal of Environmental Research and Public Health, 15(11), Article 2471. https: / / doi.org/10.3390/ijerph15112471

*Marganski, A., \& Melander, L. (2015). Intimate partner violence victimization in the cyber and real world: Examining the extent of cyber aggression experiences and its association with in-person dating violence. Journal of Interpersonal Violence, 33(7), 1071-1095. https:/ / doi.org/ $10.1177 / 0886260515614283$

*Melander, L. A., \& Marganski, A. J. (2020). Cyber and in-person intimate partner violence victimization: Examining maladaptive psychosocial and behavioral correlates. Cyberpsychology: Journal of Psychosocial Research on Cyberspace, 14, Article 1. https:/ / doi.org/10.5817/ CP2020-1-1

Mokkink, L. B., Prinsen, C. A. C., Patrick, D. L., Alonso, J., Bouter, L. M., de Vet, H. C. W., \& Terwee, C. B. (2018). COSMIN methodology for systematic reviews of Patient-Reported Outcome Measures (PROMs). https: / / www.cosmin.nl/wpcontent/uploads/COSMIN-syst-review-for-PROMsmanual_version-1_feb-2018.pdf

*Morelli, M., Bianchi, D., Chirumbolo, A., \& Baiocco, R. (2018). The cyber dating violence inventory. Validation of a new scale for online perpetration and victimization among dating partners. European Journal of Developmental Psychology, 15(4), 464-471. https: / / doi.org/10.1080/ 17405629.2017.1305885

*Muñiz Rivas, M. (2017). Violencia de pareja online en la adolescencia, clima familiar y escolar desde la perspectiva de género [Online teen dating violence, family and school climate from a gender perspective]. Journal for the Study of Education and Development, 40(3), 572-598. https: / / doi.org/ $10.1080 / 02103702.2017 .1341101$
*Muñiz Rivas, M., \& Monreal Gimeno, M. C. (2017). Violencia de pareja online y offline en la adolescencia: el rol de la soledad y del género [Online and offline teen dating violence: The role of loneliness and gender]. Revista sobre la Infancia y la Adolescencia, 9, 85-97. https:/ / doi.org/10.17060/

ijodaep.2017.n1.v2.924

*Muñoz-Fernández, N., \& Sánchez-Jiménez, V. (2020). Cyber-aggression and psychological aggression in adolescent couples: A short-term longitudinal study on prevalence and common and differential predictors. Computers in Human Behavior, 104, Article 106191. https: / / doi.org/10.1016/j.chb.2019.106191

Núñez, A., Álvarez-García, D., \& Pérez-Fuentes, M.-C. (2021). Ansiedad y autoestima en los perfiles de cibervictimización de los adolescentes [Anxiety and self-esteem in cybervictimization profiles of adolescents]. Comunicar, 67(29), 47-59. https: / / doi.org/10.3916/C67-2021-04

*Ortega-Barón, J., González-Cabrera, J., Machimbarrena, J. M., \& Montiel, I. (2021). Safety.Net: A pilot study on a Multi-Risk Internet Prevention Program. International Journal of Environmental Research and Public Health, 18(8), Article 4249. https: / / doi.org/10.3390/ijerph18084249

*Ortega-Barón, J., Montiel, I., Machimbarrena, J. M., Fernández-González, L., Calvete, E., \& González-Cabrera, J. (2020). Epidemiology of cyber dating abuse victimization in adolescence and its relationship with health-related quality of life: A longitudinal study. Youth $\mathcal{E}$ Society. Advance online publication. https:/ / doi.org/10.1177/ 0044118X20980025

Page, M. J., McKenzie, J. E., Bossuyt, P. M., Boutron, I., Hoffmann, T. C., Mulrow, C. D., Shamseer, L., Tetzlaff, J. M., Akl, E. A., Brennan, S. E., Chou, R., Glanville, J., Grimshaw, J. M., Hróbjartsson, A., Lalu, M. M., Li, T., Loder, E. W., Mayo-Wilson, E., McDonald S., ... Moher, D. (2021). The PRISMA 2020 statement: An updated guideline for reporting systematic reviews. BMJ, 372, Article 71. https: / / doi.org/10.1136/bmj.n71

*Peña Cárdenas, F., Rojas-Solís, J. L., \& García-Sánchez, P. V. (2018). Uso problemático de Internet, cyberbullying y ciberviolencia de pareja en jóvenes universitarios [Problematic Internet use, cyberbullying and cyber dating violence among university students]. Diversitas, 14(2), 205-219. http:/ / doi.org/10.15332/s1794-9998.2018.0014.01

*Picard, P. (2007). Tech Abuse in Teen Relationships Study. New York, NY: Liz Claiborne Inc.

*Preddy, T. M. (2015). Assessment and investigation of electronic aggression in the romantic relationships of emerging adults [Unpublished doctoral dissertation]. The University of Tennessee.

Prinsen, C., Mokkink, L. B., Bouter, L. M., Alonso, J., Patrick, D. L., de Vet, H., \& Terwee, C. B. (2018). COSMIN guideline for systematic reviews of patient-reported outcome measures. Quality of life research: an international journal of quality of life aspects of treatment, care and rehabilitation, 27(5), 1147-1157. https: / / doi.org/10.1007/s11136-018-1798-3

*Ramos, M. C., Miller, K. F., Moss, I. K., \& Margolin, G. (2021). Perspective-taking and empathy mitigate family-oforigin risk for electronic aggression perpetration toward dating partners: A brief report. Journal of Interpersonal 
Violence, 36(3-4), NP1155-NP1164. https: / / doi.org/ $10.1177 / 0886260517747605$

*Reed, L. A., Conn, K., \& Wachter, K. (2020). Name-calling, jealousy, and break-ups: Teen girls' and boys' worst experiences of digital dating. Children and Youth Services Review, 108, Article 104607. https:/ / doi.org/10.1016/ j.childyouth.2019.104607

*Reed, L. A., Cosgrove, J. M., Sharkey, J. D., \& Felix, E. (2020). Exploring Latinx youth experiences of digital dating abuse. Social Work Research, 44, 3, 157-168, https:/ / doi.org/ 10.1093/swr/svaa011

*Reed, L. A., Lawler, S. M., Cosgrove, J. M., Tolman, R. M., \& Ward, L. M. (2021). "It was a joke": Patterns in girls' and boys' self-reported motivations for digital dating abuse behaviors. Children and Youth Services Review, 122, Article 105883. https: / / doi.org/10.1016/j.childyouth.2020.105883

*Reed, L. A., Tolman, R. M., \& Ward, L. M. (2016). Snooping and sexting: Digital media as a context for dating aggression and abuse among college students. Violence Against Women, 22, 1556-1576. https:/ / doi.org/10.1177/1077801216630143

*Reed, L. A., Tolman, R. M., \& Ward, L. M. (2017). Gender matters: Experiences and consequences of digital dating abuse victimization in adolescent dating relationships. Journal of Adolescence, 59, 79-89. https:/ / doi.org/10.1016/ j.adolescence.2017.05.015

*Reed, L. A., Ward, M., Tolman, R. M., Lippman, J. L., \& Seabrook, R. C. (2018). The association between stereotypical gender and dating beliefs and digital dating abuse perpetration in adolescent dating relationships. Journal of Interpersonal Violence, 36, NP5561-NP5585. https: / / doi.org/10.1177/0886260518801933

Rodríguez-Domínguez, C., Pérez-Moreno, P. J., \& Durán, M. (2020). Cyber dating violence: A review of its research methodology. Anales de Psicología, 36(2), 200-209. https:/ / doi.org/10.6018/analesps.36.2.370451

*Rojas-Solís, J. L., Guzmán-Toledo, R. M., Sarquiz-García, G. C., García-Ramírez, F. D., \& Hernández-Cruz, S. (2021). Ciber-violencia en parejas de jóvenes universitarios durante la pandemia por COVID-19 [Cyber dating violence in college students during pandemic due COVID-19]. Eureka, 18(2), 227-243. https: / /n2t.net/ark:/13683/ppxs/r7c

*Romo-Tobón, R., Vázquez-Sánchez, V., Rojas-Solís, J. L., \& Alvídrez, S. (2020). Cyberbullying y ciberviolencia de pareja en alumnado de una universidad privada mexicana [Cyberbullying and cyber dating violence in students of a private Mexican university]. Propósitos y Representaciones, 8(2), Article e303. http:/ / doi.org/ 10.20511/pyr2020.v8n2.303

*Sánchez, V., Muñoz-Fernández, N., \& Ortega-Ruíz, R. (2015). "Cyber dating Q_ A": An instrument to assess the quality of adolescent dating relationships in social networks. Computers in Human Behavior, 48, 78-86. https: / / doi.org/ 10.1016/j.chb.2015.01.006

*Smith-Darden, J., Kernsmith, P. D., Victor, B. G., \& Lathrop, R. A. (2017). Electronic displays of aggression in teen dating relationships: Does the social ecology matter? Computers in Human Behavior, 67, 33-40. https:/ / doi.org/10.1016/ j.chb.2016.10.015

*Smith, K., Cénat, J. M., Lapierre, A., Dion, J., Hébert, M., \& Côte, K. (2018). Cyber dating violence: Prevalence and correlates among high school students from small urban areas in Quebec. Journal of Affective Disorders, 234, 220-223. https:/ / doi.org/10.1016/j.jad.2018.02.043

*Stewart, R. W., Drescher, C. F., Maack, D. J., Ebesutani, C., \& Young, J., (2014). The development and psychometric investigation of the Cyberbullying Scale. Journal of Interpersonal Violence, 29, 2218-2238. https:/ / doi.org/ $10.1177 \% 2 F 0886260513517552$

*Stonard, K. E. (2018). The prevalence and overlap of technology-assisted and offline adolescent dating violence. Current Psychology, 40, 1056-1070. https: / / doi.org/10.1007/ s12144-018-0023-4

*Stonard, K. E. (2020). "Technology was designed for this": Adolescents' perceptions of the role and impact of the use of technology in cyber dating violence. Computers in Human Behavior 105, Article 106211. https: / / doi.org/10.1016/ j.chb.2019.106211

*Temple, J. R., Choi, H. J., Brem, M., Wolford-Clevenger, C., Stuart, G. L., Peskin, M. F., \& Elmquist, J. (2016). The temporal association between traditional and cyber dating abuse among adolescents. Journal of Youth Adolescence 45, 340-349 https: / / doi.org/10.1007/s10964-015-0380-3

Terwee, C. B., Bot, S. D. M., de Boer, M. R., van der Windt, D. A. W. M., Knol, D. L., Dekker, J., Bouter, L. M., \& de Vet, H. C. W. (2007). Quality criteria were proposed for measurement properties of health status questionnaires Journal of Clinical Epidemiology, 60, 34-42. https: / / doi.org/ 10.1016/j.jclinepi.2006.03.012

Terwee, C. B., Prinsen, C. A. C., Chiarotto, A., Westerman, M. J., Patrick, D. L.; Alonso, J., Bouter, L. M., de Vet, H. C. W., \& Mokkink, L. B. (2018). COSMIN methodology for evaluating the content validity of patient- reported outcome measures: A Delphi Study. Quality of Life Research, 27, 1159-1170. https: / / doi.org/10.1007/s11136-018-1829-0

*Thulin, E. J., Heinze, J. E., Kernsmith, P., Smith-Darden, J., \& Fleming, P. J. (2020). Adolescent risk of dating violence and electronic dating abuse: A latent class analysis. Journal of Youth Adolescence, 50, 2472-2486. https://doi.org/10.1007/ s10964-020-01361-4

*Toplu-Demirtaș, E., May, R. W., Seibert, G. S., \& Fincham, F. D. (2020). Does cyber dating abuse victimization increase depressive symptoms or vice versa? Journal of Interpersonal Violence. Advance online publication. https:/ / doi.org/ $10.1177 / 0886260520984261$

*Toplu-Demirtaș, E., Akcabozan-Kayabol, N. B., AraciIyiaydin, A., \& Fincham, F. D. (2020). Unraveling the roles of distrust, suspicion of infidelity, and jealousy in cyber dating abuse perpetration: An attachment theory perspective. Journal of Interpersonal Violence. Advance online publication. https: / / doi.org/10.1177/0886260520927505

*van Ouytsel, J., Ponnet, K., \& Walrave, M. (2016). Cyber dating abuse victimization among secondary school students from a lifestyle-routine activities theory perspective. Journal of Interpersonal Violence, 33(17), 2767-2776. https: / / doi.org/ $10.1177 / 0886260516629390$

*van Ouytsel, J., Ponnet, K., Walrave, M., \& Temple, J. R. (2016). Adolescent cyber dating abuse victimization and its associations with substance use, and sexual behaviors. Public Health, 135, 147-151. https:/ / doi.org/10.1016/ j.puhe.2016.02.011 
*van Ouytsel, J., Torres, E., Choi, H. J., Ponnet, K., Walrave, M., \& Temple, J. R. (2016). The associations between substance use, sexual behaviors, bullying, deviant behaviors, health, and cyber dating abuse perpetration. The Journal of School Nursing, 33(2), 116-122. http: / / dx.doi.org/10.1177 / 1059840516683229

*van Ouytsel, J., Ponnet, K., \& Walrave, M. (2017). Cyber dating abuse: Investigating digital monitoring behaviors among adolescents from a social learning perspective. Journal of Interpersonal Violence, 35, 5157-5178. https: / / doi.org/ 10.1177\%2F0886260517719538

Van Ouytsel, J., Walrave, M., Ponnet, K., Willems, A.-S., \& Van Dam, M. (2019). Adolescents' perceptions of digital media's potential to elicit jealousy, conflict and monitoring behaviors within romantic relationships. Cyberpsychology: Journal of Psychosocial Research on Cyberspace, 13(3), article 3. https: / / doi.org/10.5817/CP2019-3-3

*Víllora, B., Navarro, R., \& Yubero, S. (2019a). Abuso online en el noviazgo y su relación con el abuso del móvil, la aceptación de la violencia y los mitos sobre el amor [Cyber dating abuse and its relationship with mobile phone abuse, acceptance of violence and myths about love]. Suma Psicológica, 26(1), 46-54. http:/ / doi.org/10.14349/ sumapsi.2019.v26.n1.6

*Víllora, B., Yubero, S., \& Navarro, R. (2019b). Associations between feminine gender norms and cyber dating abuse in female adults. Behavioral Sciences, 9(4), Article 35. https: / / doi.org/10.3390/bs9040035
*Víllora, B., Yubero, S., \& Navarro, R. (2019c). Cyber dating abuse and masculine gender norms in a sample of male adults. Future Internet, 11(4), Article 84. http:/ / doi.org/ 10.3390 / fi11040084

*Víllora, B., Navarro, R., \& Yubero, S. (2019d). The role of social-interpersonal and cognitive-individual factors in cyber dating victimization and perpetration: Comparing the direct, control, and combined forms of abuse. Journal of Interpersonal Violence, 36, 8559-8584. https: / / doi.org/ $10.1177 / 0886260519851172$

*Víllora, B., Yubero, S., \& Navarro, R. (2021). Subjective wellbeing among victimized university students: Comparison between cyber dating abuse and bullying victimization. Information Technology \& People, 34(1), 360-374. https: / / doi.org/10.1108/ITP-11-2018-0535

*Wright, M. F. (2015). Cyber aggression within adolescents' romantic relationships: Linkages to parental and partner attachment. Journal of Youth and Adolescence, 44, 37-47. https:/ / doi.org/10.1007/s10964-014-0147-2

*Zweig, J. M., Dank, M., Yahner, J., \& Lachman, P. (2013). The rate of cyber dating abuse among teens and how it relates to other forms of teen dating violence. Journal of Youth Adolescence, 43, 1306-1321. https: / / doi.org/10.1007/s10964013-9922-8

Zweig, J. M., Lachman, P., Yahner, J., \& Dank, M. (2014). Correlates of cyber dating abuse among teens. Journal of Youth Adolescence, 43, 1306-1321. https: / / doi.org/10.1007/ s10964-013-0047-x 


\section{Appendices}

\section{Appendix A: Studies which Have Applied some CDV Instruments but are not Psychometric Studies}

\author{
Instrument/Source \\ 1. Electronic Victimization (EV) \\ Bennett et al. (2011) \\ 2. Cyber Dating Abuse Questionnaire (CDAQ-A) \\ Borrajo, Gámez-Guadix, \& Calvete (2015a) \\ 3. Cyber Dating Abuse Questionnaire (CDAQ-B) \\ Borrajo, Gámez-Guadix, \& Calvete (2015b)
}

4. Technology-facilitated Abuse in Relationships Scale (TAR) Brown \& Hegarty (2021)

5. Controlling Partner Inventory (CPI)

Burke et al. (2011)

6. Adolescent Relationship Abuse Questionnaire (CARPA) Calvete et al. (2021)

7. Cyber Dating Abuse Scale in Adolescent Dating (ECVPA) Cava \& Buelga (2018)

8. Cyber Dating Abuse Questionnaire (CDAQ-C) Celsi et al. (2021)

9. Experience with Digital Dating Abuse (EDDA) Hinduja \& Patchin (2020)

10. Intimate Partner Violence expressed through Digital Media Scale (EVIME)

Jaen-Cortés et al. (2017)

11. Cyber Psychological Abuse Scale (CPAS)

Leisring \& Giumetti (2014)

12. Digital Intimate Partner Violence Questionnaire (DIPVQ) López-Cepero et al. (2018)

13. Cyber Aggression Measure (CAM)

Marganski \& Melander (2015)

14. The Cyber Dating Violence Inventory (TCDVI) Morelli et al. (2018)

15. Intimate Partner Violence in Social Media in Adolescents Scale (E-Vpa)

Muñiz Rivas \& Monreal Gimeno (2017)

Teen Dating Violence in Social Networks Scale (TDVSNS) Muñiz Rivas (2017)

16. Partner Electronic Aggression Questionnaire (PEAQ) Preddy (2015)

17. Digital Dating Abuse Measure (DDAM-A)

Reed et al. (2016)

Digital Dating Abuse Measure (DDAM-B)

Reed et al. (2017): Adapted from Reed et al. (2016)

18. The Cyber dating $Q \_A$ Scale (CQ_AS)

Sánchez et al. (2015)
Studies where the instrument is used

Bennett et al. (2011); Ramos et al. (2021)

Borrajo, Gámez-Guadix, \& Calvete (2015a)

Borrajo, Gámez-Guadix, \& Calvete (2015b); Borrajo, Gámez-Guadix, Pereda et al. (2015); Borrajo \& Gámez-Guadix (2016); Caridade \& Braga (2019); Caridade et al. (2020); Deans \& Bhogal (2019); Fernández-González et al. (2020); Gracia-Leiva et al. (2020); Hidalgo-Rasmussen et al. (2020); Javier-Juárez et al. (2021); Lara (2020); Machimbarrena et al. (2018); Peña Cárdenas et al. (2018); Rojas-Solís et al. (2021); Romo-Tobón et al. (2020); van Ouytsel, Ponnet \& Walrave (2016); van Ouytsel, Ponnet, Walrave \& Temple (2016); van Ouytsel, et al. (2017); Víllora et al. (2019a); Víllora et al. (2019b); Víllora et al. (2019c); Víllora et al. (2019d); Víllora et al. (2021)

Brown \& Hegarty (2021); Brown et al. (2021)

Brem, Romero et al. (2019); Burke et al. (2011)

Calvete et al. (2021); Ortega-Barón et al. (2020); Ortega-Barón et al. (2021)

Cava \& Buelga (2018); Cava, Buelga et al. (2020); Cava, MartínezFerrer et al. (2020); Cava, Tomás et al. (2020)

Celsi et al. (2021)

Hinduja \& Patchin (2020)

Jaen-Cortés et al. (2017)

Brem, Stuart et al. (2019); Leisring \& Giumetti (2014)

López-Cepero et al. (2018)

Marganski \& Melander (2015) Melander \& Marganski (2020)

Araci-İyiaydin et al. (2020); Morelli et al. (2018); Toplu-Demirtaş, Akcabozan-Kayabol et al. (2020); Toplu-Demirtaş, May et al. (2020) Muñiz Rivas \& Monreal Gimeno (2017)

Muñiz Rivas (2017)

Preddy (2015)

Bhogal \& Howman (2019); Bhogal et al. (2019); Bhogal et al. (2021); Reed et al. (2016); Reed et al. (2018)

Ellyson et al. (2021); Reed et al. (2017); Reed, Conn et al. (2020); Reed, Cosgrove et al. (2020); Reed et al. (2021)

Sánchez et al. (2015) 
Continued.

Instrument/Source

19. Cyber Dating Abuse Questionnaire (CDAQ-C) Smith et al. (2018): Adapted from cyberbullying scales

(Litwiller \& Brausch, 2013; Stewart et al., 2014)

20. Electronic Dating Aggression Survey (EDAS) Smith-Darden et al. (2017) adapted from Finkelor et al. (2000)

21. Technology Assisted Adolescent Violence (TAAV) Stonard (2018)

22. Electronic Dating Aggression (EDA)

Thulin et al. (2020)

23. Partner Directed Cyber Aggression Scale (PDCAS) Wright (2015), adapted by Linder et al. (2002)

24. Cyber Dating Abuse Questionnaire (CDAQ-D) Zweig et al. (2013) adapted from Picard (2007)
Studies where the instrument is used

Smith et al. (2018)

Smith-Darden et al. (2017)

Stonard (2018); Stonard (2020)

Thulin et al. (2020)

Wright (2015)

Dank et al. (2013); Dyar et al. (2020); Lu et al. (2018); Lu et al. (2020); Muñoz-Fernández \& Sánchez-Jiménez (2020); Temple et al. (2016); van Ouytsel, Torres, et al. (2016); Zweig et al. (2013)

\section{Appendix B}

\begin{tabular}{|c|c|c|}
\hline Property & Definition & When it is considered positive \\
\hline $\begin{array}{l}\text { Content } \\
\text { Validity }\end{array}$ & $\begin{array}{l}\text { Extent to which a measure represents all facets of a } \\
\text { given construct, when it is an adequate reflection of } \\
\text { the construct. }\end{array}$ & $\begin{array}{l}\text { When a clear description is provided of the } \\
\text { measurement aim, the target population, the } \\
\text { concepts that are being measured, and the item } \\
\text { selection AND target population and investigators } \\
\text { are involved in item selection }\end{array}$ \\
\hline $\begin{array}{l}\text { Structural } \\
\text { Validity }\end{array}$ & $\begin{array}{l}\text { The degree to which the scores of an instrument are an } \\
\text { adequate reflection of the dimensionality of the } \\
\text { construct to be measured. }\end{array}$ & $\begin{array}{l}\text { When data of exploratory and/or confirmatory } \\
\text { factorial analysis: RMSEA <.08-.05; CFI, NNFI > } \\
.90-.95\end{array}$ \\
\hline $\begin{array}{l}\text { Cross-cultural } \\
\text { Validity }\end{array}$ & $\begin{array}{l}\text { The degree to which the performance of the items on a } \\
\text { translated or culturally adapted instrument are an } \\
\text { adequate reflection of the performance of the items } \\
\text { of the original version of the instrument. }\end{array}$ & $\begin{array}{l}\text { When there are no important differences between } \\
\text { group factors in trans-cultural studies. }\end{array}$ \\
\hline $\begin{array}{l}\text { Hypotheses } \\
\text { testing }\end{array}$ & $\begin{array}{l}\text { The degree to which the scores of an instrument are } \\
\text { consistent with hypotheses. }\end{array}$ & $\begin{array}{l}\text { When the result is in accordance with the hypotheses } \\
\text { (specific hypotheses are formulated AND at least } \\
75 \% \text { of the results are in accordance with these } \\
\text { hypotheses). }\end{array}$ \\
\hline Reliability & $\begin{array}{l}\text { The extent to which patients can be distinguished } \\
\text { from each other, despite measurement errors } \\
\text { (relative measurement error). }\end{array}$ & When: ICC or Kappa > .70 \\
\hline $\begin{array}{l}\text { Internal } \\
\text { Consistency }\end{array}$ & $\begin{array}{l}\text { The extent to which items in a (sub)scale are } \\
\text { intercorrelated, thus measuring the same construct. }\end{array}$ & $\begin{array}{l}\text { When factor analyses are performed on adequate } \\
\text { sample size AND Cronbach's alpha(s) are } \\
\text { calculated per dimension AND Cronbach's alpha(s) } \\
\text { are between } .70 \text { and } .95 \text {. }\end{array}$ \\
\hline $\begin{array}{l}\text { Criterion } \\
\text { Validity }\end{array}$ & $\begin{array}{l}\text { The extent to which scores on a particular } \\
\text { questionnaire relate to a gold standard. }\end{array}$ & Correlation with gold standard $\geq .70$ OR AUC $\geq .70$. \\
\hline Responsiveness & $\begin{array}{l}\text { The ability of a questionnaire to detect clinically } \\
\text { important changes over time. }\end{array}$ & $\begin{array}{l}\text { The result is in accordance with the hypothesis OR } \\
\text { AUC } \geq .70 \text {. }\end{array}$ \\
\hline
\end{tabular}

